\title{
A Hybrid Design Approach for Output Feedback Exponential Stabilization of Markovian Jump Systems
}

\author{
Jun Song, Yugang Niu, James Lam, Fellow, IEEE, and Zhan Shu, Member, IEEE
}

\begin{abstract}
This work deals with the exponential stabilization problem of discrete-time Markovian jump systems via a hybrid control strategy, in which the transition probability matrix and static output-feedback controller are designed simultaneously. A necessary and sufficient condition for the existence of an exponential stabilizing transition probability matrix is derived by means of a mode-dependent parametric approach. Furthermore, a sufficient condition is established for the above hybrid design with a specified lower bound on the decay rate. The proposed design approaches can be applied to solve two kinds of control design problems with practical constraints imposed on the hybrid design. Besides, an estimation approach is proposed on the decay rate and decay coefficient of the jump systems. Also, two optimization problems are formulated to obtain the hybrid control strategy. Finally, two numerical examples and a networkon-chip (NoC) based application are provided to illustrate the effectiveness of the proposed results.
\end{abstract}

Index Terms-Discrete time, Exponential stability, Markovian jump systems, Transition probability

\section{INTRODUCTION}

$\mathbf{M}$ ARKOVIAN jump linear systems (MJLSs) have received considerable attention since they may effectively represent plants with abrupt variations in their structures, such as random failures of components, sudden environmental disturbances, changing subsystems interconnections, and abrupt variations in the operating point of a nonlinear plant. Some relevant results on the stability, stabilization, and optimal control of MJLSs can be found in [1]-[7], and the references therein.

However, most of the existing synthesis approaches for MJLSs are based on the assumption that the Markov transition matrices are fixed a priori. Apparently, this assumption may not be valid in some practical cases, in which engineers may have the freedom to choose/design appropriate Markov transition matrices or general switching rules, since an appropriate switching rule may stabilize a large-scale system with none of

This work was supported in part by the National Natural Science Foundation of China (61673174, 61773162), Cheung Kong Chair Professor Program, GRF (17205815), and the 111 Project (B17017) from China. (Corresponding authors: Yugang Niu and James Lam)

J. Song and Y. Niu are with the Key Laboratory of Advanced Control and Optimization for Chemical Process (East China University of Science and Technology), Ministry of Education, Shanghai 200237, China (e-mail: sjky5211@163.com; acniuyg@ecust.edu.cn).

J. Lam is with the Department of Mechanical Engineering, The University of Hong Kong, Pokfulam Road, Hong Kong, China (e-mail: james.lam@hku.hk).

Z. Shu is with the School of Engineering Sciences, University of Southampton, Southampton SO17 1BJ, U.K. (e-mail: z.shu@ soton.ac.uk). the individual subsystems asymptotically stable [8]. An early work on the probability rate synthesis of MJLSs can be found in [9], wherein a necessary and sufficient characterization of stabilizing transition rate matrices for continuous-time MJLSs was established based on linear programming. Following this work, a sufficient condition for designing desired controller gains and transition rate matrices was provided for continuoustime MJLSs with time-delays in [10] and guaranteed cost stabilization problem via switching probability rate synthesis was also given in [11]. However, to date, there is little research on the hybrid design for discrete-time Markovian jump systems. It is worth pointing out that the discrete-time case cannot be solved trivially by adopting the existing continuous-time approaches. This is because when the transition probability matrix is considered as a decision variable, the discretetime coupled algebraic Riccati matrix inequality will contain one 4-degree and two 3-degree coupling terms, which shows significantly difference from the continuous-time one (see Subsection III-B for more details).

Recently, Sehgal in [12] carried out an interesting research on a class of stochastic communication in networks-in-package in which there may exist latency, duration of active routers for power saving, lost data packets, and their exact location for dynamic buffer allocation during parallel communication. A probabilistic communication scheme via Markov jump model was proposed in [12]. Moreover, it was also shown that some desired but highly structured discrete-time Markov transition probability matrices (for example, Gossip Markov Chain) could be mapped on the corresponding Network-onChip (NoC) by adjusting the data packet flow rates and the time between events or trials. These engineering techniques have also motivated us to consider, for a general discretetime Markovian jump systems, how to synthesize the transition probability matrix under multiple constraints? Moreover, what relations may there be between the transition probability matrix and the controller for improving system stability? These issues remain open still.

In this work, we consider the exponential stabilization problem for discrete-time MJLSs via a hybrid control strategy, in which the transition probability matrix and the modedependent static output feedback (MDSOF) controller are synthesized simultaneously. By introducing a mode-dependent parametric approach, a necessary and sufficient condition is established for the existence of transition probability matrices that render the exponential mean-square stability (EMSS) with a specified lower bound $\gamma$ on the decay rate. Then, a sufficient 
condition for MDSOF synthesis is proposed. Moreover, the hybrid designs under gain constraints and structured transition probability matrix are addressed and two optimization formulations are proposed to obtain the desired hybrid control strategy.

The application prospect of this work includes the following two kinds of control design problems:

(1) The first kind of problem was initiated in [8] (see Problem C therein), and further addressed for the continuoustime MJLSs in [9]. This work solves this kind of problem for discrete-time case. Indeed, the following two subproblems are summarized.

- Problem 1a. When none of the individual subsystems is asymptotically stable, design a stabilizing transition probability matrix $\Pi$ such that the reconstructed discretetime MJLS is EMSS for a given scalar $\gamma$.

- Problem 1b. When none of the individual subsystems is asymptotically stable and output controllable by single SOF, find a stabilizing transition probability matrix $\Pi$ and MDSOF controller $K_{i}$ such that the closed-loop MJLS is EMSS for a given scalar $\gamma$.

(2) The second kind of problem is for the case that at least one of the individual subsystems is asymptotically stable. It should be pointed out that in this case, Problem C in [8] and [9] will become trivial. For example, we consider two discretetime subsystems $A_{1}$ and $A_{2}$. The former is asymptotically stable but the latter is not. If one just wants to stabilize the MJLS composed of $A_{1}$ and $A_{2}$, as pointed out in [8], a stabilizing transition probability matrix can be readily chosen as $\Pi=\left[\begin{array}{lll}1 & 0 ; 1 & 0\end{array}\right]$. That will make the reconstructed discretetime MJLS share the same decay rate as the subsystem $A_{1}$. However, some practical applications may need a larger decay rate for the reconstructed MJLSs than subsystem $A_{1}$. This fact motivates us to consider the following problems:

- Problem 2a. In the case that at least one of the individual subsystems is asymptotically stable, design a transition probability matrix $\Pi$ such that the discrete-time MJLS has a decay rate larger than this stable subsystem.

- Problem 2b. In the case that at least one of the individual subsystems is asymptotically stable and none of them is output controllable by single SOF, find a transition probability matrix $\Pi$ and MDSOF controller $K_{i}$ such that the closed-loop MJLS has a decay rate larger than this stable subsystem.

In other words, by designing a suitable hybrid control strategy, some 'slow' and 'unstable' subsystems under a deterministic setting can be formulated into a 'fast' MJLS under a stochastic setting, which is a particularly interesting application area of this work.

Notation. Throughout this paper, if not explicitly stated, matrices are assumed to have compatible dimensions. $(\Omega, \mathcal{F}, \operatorname{Pr})$ is a probability space with $\Omega$ the sample space, $\mathcal{F}$ is the $\sigma$-algebra of subsets of the sample space, and $\operatorname{Pr}$ is the probability measure. $\mathbf{E}\{\cdot\}$ denotes the expectation operator with respect to probability measure $\operatorname{Pr} . \operatorname{diag}\{\cdot\}$ stands for a block-diagonal matrix. " $\star$ " is used to represent the elements below the main diagonal of a symmetric block matrix. The notation $A \succeq 0$ means that $A$ is a nonnegative matrix, that is, all elements in $A$ are nonnegative. $\rho(A)$ denotes the spectral radius of matrix $A$. "( $\left.\Pi, K_{i}\right)$ " denotes the hybrid control strategy, that is, the transition probability matrix $\Pi$ and the MDSOF controller $K_{i}$ are employed simultaneously.

\section{Problem Formulation and Preliminaries}

For the probability space $(\Omega, \mathcal{F}, \operatorname{Pr})$, consider the following discrete-time MJLS:

$$
\begin{aligned}
x(k+1) & =A\left(r_{k}\right) x(k)+B\left(r_{k}\right) u(k), \\
y(k) & =C\left(r_{k}\right) x(k),
\end{aligned}
$$

where $x(k) \in \mathbb{R}^{n}, u(k) \in \mathbb{R}^{m}$, and $y(k) \in \mathbb{R}^{p}$ are the state, control input, and the measured output, respectively. The jumping process $\left\{r_{k} \mid k \in \mathbb{N}_{0}\right\}$ is described by a discrete-time homogeneous Markov chain, which takes values in a finite set $\mathcal{L} \triangleq\{1,2, \ldots, N\}$ with mode transition probabilities:

$$
\operatorname{Pr}\left(r_{k+1}=j \mid r_{k}=i\right)=\pi_{i j}
$$

where $1 \geq \pi_{i j} \geq 0, \forall i, j \in \mathcal{L}$, and $\sum_{j=1}^{N} \pi_{i j}=1$. The set $\mathcal{L}$ indexes the $N$ modes of system (1)-(2) and for $r_{k}=i$, the system matrices of the $i$ th mode are denoted by $A_{i}, B_{i}$ and $C_{i}$. Note that the transition probability matrix $\Pi \triangleq\left[\pi_{i j}\right]_{N \times N}$ is a nonnegative matrix, that is, $\Pi \succeq 0$.

For the underlying system, the following definition and lemma will be adopted in this work. For more details, readers are referred to [13]-[16] and the references therein.

Definition 1: For a scalar $\gamma \geq 1$, system (1) is said to be $\gamma$ exponentially mean-square stable ( $\gamma$-EMSS) if, for $u(k) \equiv 0$, any given initial condition $x_{0} \in \mathbb{R}^{n}$ and initial mode $r_{0} \in \mathcal{L}$, there exist scalars $b>0$ and $\sigma>0$ such that for all $k \in \mathbb{N}_{+}$,

$$
\mathbf{E}\left\{\|x(k)\|^{2} \mid x_{0}, r_{0}\right\} \leq b(\gamma+\sigma)^{-k}\left\|x_{0}\right\|^{2},
$$

where $\gamma+\sigma$ and $b$ are the decay rate and decay coefficient, respectively.

Lemma 1: For a given transition probability matrix $\Pi \succeq 0$, unforced system (1) is $\gamma$-EMSS if and only if there exists a set of positive definite matrices $P_{i}, i \in \mathcal{L}$, satisfying the following inequalities:

$$
\gamma A_{i}^{T}\left(\sum_{j=1}^{N} \pi_{i j} P_{j}\right) A_{i}-P_{i}<0, i \in \mathcal{L} .
$$

In this work, it is assumed that the transition probability matrix $\Pi$ may be designed or changed by some physical techniques as described in [12], [17]. The first task of this work is to establish a necessary and sufficient condition for synthesizing a transition probability matrix $\Pi$ such that the unforced system (1) is $\gamma$-EMSS. The second is to design a mode-dependent static output-feedback (MDSOF) controller as

$$
u(k)=K_{i} y(k)=K_{i} C_{i} x(k), i \in \mathcal{L},
$$

and a transition probability matrix $\Pi$ such that the resultant closed-loop system is $\gamma$-EMSS with a decay rate larger than a specified scalar $\gamma \geq 1$. Here, there are $N^{2}$ scalars $\pi_{i j}$ and $N$ matrices $K_{i}$ to be determined in the above hybrid design. 


\section{Main Results}

\section{A. Stabilizing Transition Probability Matrices}

In the sequel, we shall investigate the stochastic stabilizing problem of the unforced system in (1), that is, $u(k) \equiv 0$, via transition probability synthesis. Inspired by the ideas in [9], [18], [19], the following necessary and sufficient condition is derived for synthesizing a stabilizing transition probability matrix $\Pi$.

Theorem 1: For a given scalar $\gamma \geq 1$, there exists a transition probability matrix $\Pi$ such that the unforced system (1) is $\gamma$-EMSS if and only if for a set of given scalars $\epsilon_{i}>0$, $i \in \mathcal{L}$, there exist two sets of positive definite matrices $\left\{X_{i}\right\}$ and $\left\{\bar{X}_{i}\right\}$, and a matrix $\Pi \succeq 0$ satisfying the following inequalities:

$$
\begin{gathered}
{\left[\begin{array}{cc}
-\gamma^{-1} X_{i}-\epsilon_{i}^{-1} \sum_{\star=1}^{N} A_{i}^{T} X_{j} A_{i} & \Phi_{i} \\
\star & -\bar{\Lambda}_{i}
\end{array}\right]<0,} \\
\operatorname{rank}\left(\left[\begin{array}{cc}
X_{i} & I \\
I & \bar{X}_{i}
\end{array}\right]\right) \leq n
\end{gathered}
$$

where

$$
\begin{aligned}
& \Phi_{i} \triangleq\left[A_{i}^{T}+\frac{1}{2} \epsilon_{i} \pi_{i 1} A_{i}^{T} \quad A_{i}^{T}+\frac{1}{2} \epsilon_{i} \pi_{i 2} A_{i}^{T}\right. \\
& \text { ‥ } \left.A_{i}^{T}+\frac{1}{2} \epsilon_{i} \pi_{i N} A_{i}^{T}\right], \\
& \bar{\Lambda}_{i} \triangleq \operatorname{diag}\left\{\epsilon_{i} \bar{X}_{1}, \epsilon_{i} \bar{X}_{2}, \ldots, \epsilon_{i} \bar{X}_{N}\right\} .
\end{aligned}
$$

Moreover, a stabilizing transition probability matrix is given by $\Pi$.

Proof: For any mode $i \in \mathcal{L}$, inequality (5) holds if and only if the following inequality holds for a sufficiently small scalar $\epsilon_{i}>0$ :

$$
\begin{gathered}
-P_{i}+\gamma \sum_{j=1}^{N}\left(\frac{1}{2} \pi_{i j} A_{i}\right)^{T} P_{j} A_{i}+\gamma \sum_{j=1}^{N} A_{i}^{T} P_{j}\left(\frac{1}{2} \pi_{i j} A_{i}\right) \\
+\epsilon_{i} \gamma \sum_{j=1}^{N}\left(\frac{1}{2} \pi_{i j} A_{i}\right)^{T} P_{j}\left(\frac{1}{2} \pi_{i j} A_{i}\right)<0
\end{gathered}
$$

By Schur complement equivalence, it is easily shown that the inequalities (9) hold if and only if the following inequalities hold:

$$
\left[\begin{array}{cc}
-P_{i}-\frac{\gamma}{\epsilon_{i}} \sum_{j=1}^{N} A_{i}^{T} P_{j} A_{i} & \Phi_{i} \\
\star & -\Lambda_{i}
\end{array}\right]<0, \forall i \in \mathcal{L},
$$

with $\Lambda_{i} \triangleq \operatorname{diag}\left\{\frac{\epsilon_{i}}{\gamma} P_{1}^{-1}, \frac{\epsilon_{i}}{\gamma} P_{2}^{-1}, \ldots, \frac{\epsilon_{i}}{\gamma} P_{N}^{-1}\right\}$.

Define $X_{i}=\gamma P_{i}$ and $\bar{X}_{i}=\left(\gamma P_{i}\right)^{-1}$, the inequalities in (10) can be reformulated as the inequalities in (7) with matrix equality constraints $X_{i} \bar{X}_{i}=I$. As pointed out in [10], [20], these matrix equality constraints are equivalent to the rank constraints in (8). This completes the proof.

Remark 1: The key idea of the common parameter approach in [9] is to introduce a sufficiently small modeindependent scalar $\varepsilon>0$ into some coupled Lyapunov inequalities [19]. It is noted that the conditions obtained in [9] are solvable only when the parameter $\varepsilon$ satisfies all modes. However, if for some modes $i, j \in \mathcal{L}$, the parameter $\varepsilon$ cannot satisfy the obtained conditions in [9] simultaneously, the concerned problem will become infeasible. In order to avoid the above undesirable case, a set of mode-dependent sufficiently small scalars $\epsilon_{i}>0$ are introduced in the inequality (9), which can ensure the obtained conditions in this work to find the feasible solutions more effectively than the ones derived by common parameter approach in [9]-[11]. Numerical examples in Section V also illustrate this case.

Remark 2: As a stronger version of Problem 2a, Theorem 1 can also be used to design an MJLS with a faster decay rate under a stochastic setting than any stable subsystems under a deterministic setting through modifying the transition probability matrix.

\section{B. Hybrid Design With MDSOF}

Now, we consider the problem of designing a stabilizing MDSOF controller (6) and a corresponding transition probability matrix $\Pi$ for discrete-time MJLSs (1). First, applying controller (6) to system (1) yields the following closed-loop system:

$$
x(k+1)=\left(A_{i}+B_{i} K_{i} C_{i}\right) x(k) .
$$

According to Lemma 1, the closed-loop system (11) is $\gamma$ EMSS if and only if, for a given scalar $\gamma \geq 1$, there exist a set of positive definite matrices $\left\{P_{i}\right\}$ satisfying the following inequalities:

$$
\gamma\left(A_{i}+B_{i} K_{i} C_{i}\right)^{T}\left(\sum_{j=1}^{N} \pi_{i j} P_{j}\right)\left(A_{i}+B_{i} K_{i} C_{i}\right)-P_{i}<0,
$$

that is, for any $i \in \mathcal{L}$,

$$
\begin{aligned}
& -P_{i}+\gamma A_{i}^{T}\left(\sum_{j=1}^{N} \pi_{i j} P_{j}\right) A_{i} \\
& +\gamma\left(B_{i} K_{i} C_{i}\right)^{T}\left(\sum_{j=1}^{N} \pi_{i j} P_{j}\right)\left(B_{i} K_{i} C_{i}\right) \\
& +\gamma A_{i}^{T}\left(\sum_{j=1}^{N} \pi_{i j} P_{j}\right)\left(B_{i} K_{i} C_{i}\right) \\
& +\gamma\left(B_{i} K_{i} C_{i}\right)^{T}\left(\sum_{j=1}^{N} \pi_{i j} P_{j}\right) A_{i}<0 .
\end{aligned}
$$

Obviously, if the matrices $K_{i}, P_{i}$, and the transition probability matrix $\Pi$ are considered as decision variables simultaneously for discrete-time MJLSs, there exist one 4-degree coupling $^{1}$ term, $\gamma\left(B_{i} K_{i} C_{i}\right)^{T}\left(\sum_{j=1}^{N} \pi_{i j} P_{j}\right)\left(B_{i} K_{i} C_{i}\right)$, and two 3-degree coupling terms, $\gamma A_{i}^{T}\left(\sum_{j=1}^{N} \pi_{i j} P_{j}\right)\left(B_{i} K_{i} C_{i}\right)$ and $\gamma\left(B_{i} K_{i} C_{i}\right)^{T}\left(\sum_{j=1}^{N} \pi_{i j} P_{j}\right) A_{i}$, in (13), which just shows the significant difference from the state-feedback (or static outputfeedback) stabilization of continuous-time MJLSs in [9]-[11]. Actually, for the continuous-time case, there are only 2-degree coupling (or bilinear) terms in the coupled algebra Riccati matrix inequalities (see Eq. (16) in [9] for example). This

\footnotetext{
${ }^{1}$ 'Degree of coupling' refers to the number of multiplications of the decision variables in an expression.
} 
difference results in that the approach in [9] cannot be used in the discrete-time case and the hybrid design in this work becomes more complex and challenging.

Now, we will deal with the MDSOF stabilization with transition probability synthesis for system (1).

Theorem 2: For a given scalar $\gamma \geq 1$, if for a prescribed scalar $\mu>0$ and a set of given scalars $\epsilon_{i}>0, i \in \mathcal{L}$, there exist two sets of positive definite matrices $\left\{X_{i}\right\}$ and $\left\{\bar{X}_{i}\right\}$, a set of real matrices $\left\{K_{i}\right\}$, and a matrix $\Pi \succeq 0$ satisfying the following inequalities:

$$
\begin{gathered}
{\left[\begin{array}{cccc}
\Omega_{i} & \Phi_{i} & \Xi_{i} & \Psi_{i} \\
\star & -\bar{\Lambda}_{i} & 0 & 0 \\
\star & \star & -\bar{\Upsilon} & 0 \\
\star & \star & \star & -\Gamma
\end{array}\right]<0,} \\
\operatorname{rank}\left(\left[\begin{array}{cc}
X_{i} & I \\
I & \bar{X}_{i}
\end{array}\right]\right) \leq n,
\end{gathered}
$$

where $\Phi_{i}, \bar{\Lambda}_{i}$ are defined in Theorem 1 , and

$$
\begin{aligned}
\Omega_{i} \triangleq & -\gamma^{-1} X_{i}-\epsilon_{i}^{-1} \sum_{j=1}^{N} A_{i}^{T} X_{j} A_{i}-2 \mu A_{i}^{T} A_{i}, \\
\Xi_{i} \triangleq & {\left[B_{i} K_{i} C_{i}\right)^{T}+\pi_{i 1} A_{i}^{T} \quad\left(B_{i} K_{i} C_{i}\right)^{T}+\pi_{i 2} A_{i}^{T} } \\
& \left.\ldots\left(B_{i} K_{i} C_{i}\right)^{T}+\pi_{i N} A_{i}^{T}\right],
\end{aligned}
$$

$\bar{\Upsilon} \triangleq \operatorname{diag}\left\{\bar{X}_{1}, \bar{X}_{2}, \cdots, \bar{X}_{N}\right\}, \Gamma \triangleq \operatorname{diag}\left\{X_{1}, X_{2}, \cdots, X_{N}\right\}$, $\Psi_{i} \triangleq \underbrace{\left[\begin{array}{llll}\mu A_{i}^{T} & \mu A_{i}^{T} & \cdots & \mu A_{i}^{T}\end{array}\right]}_{N}$

then the closed-loop system (11) is $\gamma$-EMSS.

Proof: Notice that for any modes $i, j \in \mathcal{L}$, it has $\sum_{i=1}^{N} \pi_{i j} P_{j} \leq \sum_{i=1}^{N} P_{j}$ due to $P_{j}>0$ and $\pi_{i j} \leq 1$. Thus, the inequality in (12) or (13) holds if there exists a sufficiently small scalar $\epsilon_{i}>0$ such that the following inequality holds:

$$
\begin{aligned}
& -P_{i}+\gamma \sum_{j=1}^{N}\left(\frac{1}{2} \pi_{i j} A_{i}\right)^{T} P_{j} A_{i}+\gamma \sum_{j=1}^{N} A_{i}^{T} P_{j}\left(\frac{1}{2} \pi_{i j} A_{i}\right) \\
& +\gamma \sum_{j=1}^{N}\left(\pi_{i j} A_{i}\right)^{T} P_{j}\left(B_{i} K_{i} C_{i}\right)+\gamma \sum_{j=1}^{N}\left(B_{i} K_{i} C_{i}\right)^{T} P_{j}\left(\pi_{i j} A_{i}\right) \\
& +\gamma\left(B_{i} K_{i} C_{i}\right)^{T}\left(\sum_{j=1}^{N} P_{j}\right)\left(B_{i} K_{i} C_{i}\right) \\
& +\epsilon_{i} \gamma \sum_{j=1}^{N}\left(\frac{1}{2} \pi_{i j} A_{i}\right)^{T} P_{j}\left(\frac{1}{2} \pi_{i j} A_{i}\right)<0
\end{aligned}
$$

It is easily shown that the inequalities in (16) are equivalent to the following inequalities:

$$
\left[\begin{array}{ccc}
\Theta_{i} & \Phi_{i} & \Xi_{i} \\
\star & -\Lambda_{i} & 0 \\
\star & \star & -\Upsilon
\end{array}\right]<0, i \in \mathcal{L},
$$

where $\Phi_{i}$ is given as in Theorem $1, \Lambda_{i}$ is defined in (10), and

$$
\begin{aligned}
\Upsilon & \triangleq \operatorname{diag}\left\{\left(\gamma P_{1}\right)^{-1},\left(\gamma P_{2}\right)^{-1}, \ldots,\left(\gamma P_{N}\right)^{-1}\right\}, \\
\Theta_{i} & \triangleq-P_{i}-\gamma \epsilon_{i}^{-1} \sum_{j=1}^{N} A_{i}^{T} P_{j} A_{i}
\end{aligned}
$$

$$
-\gamma \sum_{j=1}^{N}\left(\pi_{i j} A_{i}\right)^{T} P_{j}\left(\pi_{i j} A_{i}\right) .
$$

Noting $P_{j}>0$, we have for an adjustable scalar $\mu>0$ and any $i, j \in \mathcal{L}$,

$$
\begin{aligned}
& {\left[\pi_{i j} I-\mu\left(\gamma P_{j}\right)^{-1}\right]\left(\gamma P_{j}\right)\left[\pi_{i j} I-\mu\left(\gamma P_{j}\right)^{-1}\right] } \\
= & \mu^{2}\left(\gamma P_{j}\right)^{-1}-2 \mu \pi_{i j} I+\pi_{i j}\left(\gamma P_{j}\right) \pi_{i j} \geq 0,
\end{aligned}
$$

which gives

- $\left(\pi_{i j} A_{i}\right)^{T}\left(\gamma P_{j}\right)\left(\pi_{i j} A_{i}\right) \leq \mu^{2} A_{i}^{T}\left(\gamma P_{j}\right)^{-1} A_{i}-2 \mu \pi_{i j} A_{i}^{T} A_{i}$.

Owing to the fact that $\sum_{j=1}^{N} \pi_{i j} A_{i}^{T} A_{i}=A_{i}^{T} A_{i}$, we obtain from (19) that the each inequality in (17) can be guaranteed by the following inequalities:

$$
\left[\begin{array}{ccc}
\tilde{\Omega}_{i} & \Phi_{i} & \Xi_{i} \\
\star & -\Lambda_{i} & 0 \\
\star & \star & -\Upsilon
\end{array}\right]<0, \forall i \in \mathcal{L},
$$

where

$$
\begin{aligned}
\tilde{\Omega}_{i} \triangleq & -P_{i}-\epsilon_{i}^{-1} \gamma \sum_{j=1}^{N} A_{i}^{T} P_{j} A_{i} \\
& +\mu^{2} \sum_{j=1}^{N} A_{i}^{T}\left(\gamma P_{j}\right)^{-1} A_{i}-2 \mu A_{i}^{T} A_{i} .
\end{aligned}
$$

Next, similar to Theorem 1, define $X_{i}=\gamma P_{i}$ and $\bar{X}_{i}=$ $\left(\gamma P_{i}\right)^{-1}$, we obtain from (20) that the inequalities in (12) are satisfied if the inequalities (14)-(15) are feasible.

Remark 3: It should be noted that Theorems 1 and 2 are proved by utilizing "completing the square". Therefore, the term, $\epsilon_{i} \gamma \sum_{j=1}^{N}\left(\frac{1}{2} \pi_{i j} A_{i}\right)^{T} P_{j}\left(\frac{1}{2} \pi_{i j} A_{i}\right)$, is added into (9) and (16). On the other hand, $\pi_{i j} \leq 1$ is employed first to reduce the 4-degree coupling term into a suitable 3-degree one, $\gamma\left(B_{i} K_{i} C_{i}\right)^{T}\left(\sum_{j=1}^{N} P_{j}\right)\left(B_{i} K_{i} C_{i}\right)$. Thus, another quadratic term with respect to $B_{i} K_{i} C_{i}+\pi_{i j} A_{i}$ can be constructed in (16). Furthermore, the remaining 3-degree coupling term, $-\gamma \sum_{j=1}^{N}\left(\pi_{i j} A_{i}\right)^{T} P_{j}\left(\pi_{i j} A_{i}\right)$, in (17) is handled by employing inequality (19), where the adjustable scalar $\mu$ is introduced to enhance the feasibility of the conditions (14)-(15). Obviously, the solution algorithm for hybrid design problem of discrete-time MJLS is more complex than the continuoustime case as in [9], since mode-dependent scalars $\epsilon_{i}$ should be fixed to solve Theorem 2. Nevertheless, the difficulties in choosing $\epsilon_{i}$ will be overcome by using a gridding technique (see (61)-(62)).

\section{Necessary Conditions of The Proposed Design Methodol- ogy}

This subsection provides necessary conditions for the results in Theorems 1 and 2. Firstly, the following proposition gives a necessary condition for the proposed design methodology in Theorem 1.

Proposition 3: If inequalities (7)-(8) in Theorem 1 hold, then

$$
A_{i}^{T}\left[\gamma\left(\pi_{i i}+\frac{\epsilon_{i}}{4} \pi_{i i}^{2}\right) X_{i}\right] A_{i}<X_{i}
$$


which is equivalent to

$$
\max _{\epsilon_{i}}\left\{\pi_{i i}\right\} \leq \min _{\epsilon_{i}}\left\{g\left(\epsilon_{i}\right), 1\right\}
$$

where

$$
g\left(\epsilon_{i}\right) \triangleq \frac{2}{\gamma \rho^{2}\left(A_{i}\right)\left[\sqrt{1+\frac{\epsilon_{i}}{\gamma \rho^{2}\left(A_{i}\right)}}+1\right]} .
$$

Proof: As shown in the proof of Theorem 1, the conditions (7)-(8) is equivalent to (9), from which one has

$$
\begin{aligned}
& -P_{i}+\gamma \sum_{j=1}^{N} \pi_{i j} A_{i}^{T} P_{j} A_{i}+\epsilon_{i} \gamma \sum_{j=1}^{N}\left(\frac{1}{2} \pi_{i j} A_{i}\right)^{T} \\
& \times P_{j}\left(\frac{1}{2} \pi_{i j} A_{i}\right) \\
& =-P_{i}+\sum_{j=1}^{N} \gamma\left(1+\frac{\epsilon_{i}}{4} \pi_{i j}\right) \pi_{i j} A_{i}^{T} P_{j} A_{i} \\
& =-P_{i}+\gamma\left(\pi_{i i}+\frac{\epsilon_{i}}{4} \pi_{i i}^{2}\right) A_{i}^{T} P_{i} A_{i} \\
& \quad+\sum_{j=1, j \neq i}^{N} \gamma\left(1+\frac{\epsilon_{i}}{4} \pi_{i j}\right) A_{i}^{T} \pi_{i j} P_{j} A_{i}<0, \forall i \in \mathcal{L} .
\end{aligned}
$$

Obviously, a necessary condition for (24) to hold can be given as

$$
-P_{i}+\gamma\left(\pi_{i i}+\frac{\epsilon_{i}}{4} \pi_{i i}^{2}\right) A_{i}^{T} P_{i} A_{i}<0 .
$$

Thus, the necessary condition (21) is obtained.

Furthermore, it is noted that a necessary and sufficient condition for (25) to hold is

$$
\pi_{i i}+\frac{\epsilon_{i}}{4} \pi_{i i}^{2}<\frac{1}{\gamma \rho^{2}\left(A_{i}\right)}
$$

By (26), we have

$$
\begin{aligned}
\pi_{i i} & <\frac{2}{\epsilon_{i}}\left[\sqrt{1+\frac{\epsilon_{i}}{\gamma \rho^{2}\left(A_{i}\right)}}-1\right] \\
& =\frac{2}{\gamma \rho^{2}\left(A_{i}\right)\left[\sqrt{1+\frac{\epsilon_{i}}{\gamma \rho^{2}\left(A_{i}\right)}}+1\right]} .
\end{aligned}
$$

Combining the fact of $0 \leq \pi_{i j} \leq 1$, one can conclude from (25) and (27) that the relationship in (22) is a necessary condition for Theorem 1 to hold.

A necessary condition for Theorem 2 to hold is derived as follows.

Proposition 4: If inequalities (14)-(15) in Theorem 2 hold for a prescribed scalar $\mu>0$, then

$A_{i}^{T}\left[\gamma\left(\pi_{i i}+\frac{\epsilon_{i}}{4} \pi_{i i}^{2}\right) X_{i}+\gamma \mu^{2} \bar{X}_{i}-2 \gamma \mu I\right] A_{i}<X_{i}, \forall \epsilon_{i}>0$.

Proof: By Schur complement equivalence, it is seen that the inequality (20) or (14)-(15) is equivalent to

$$
\begin{aligned}
& -P_{i}+\mu^{2} \sum_{j=1}^{N} A_{i}^{T}\left(\gamma P_{j}\right)^{-1} A_{i}-2 \mu A_{i}^{T} A_{i} \\
& +\sum_{j=1}^{N} \gamma\left(1+\frac{\epsilon_{i}}{4} \pi_{i j}\right) \pi_{i j} A_{i}^{T} P_{j} A_{i}+\Xi_{i} \Gamma \Xi_{i}^{T}
\end{aligned}
$$

$$
\begin{aligned}
= & -P_{i}-2 \mu A_{i}^{T} A_{i}+\gamma\left(1+\frac{\epsilon_{i}}{4} \pi_{i i}\right) \pi_{i i} A_{i}^{T} P_{i} A_{i} \\
& +\mu^{2} A_{i}^{T}\left(\gamma P_{i}\right)^{-1} A_{i}+\mu^{2} \sum_{j=1, j \neq i}^{N} A_{i}^{T}\left(\gamma P_{j}\right)^{-1} A_{i} \\
& +\sum_{j=1, j \neq i}^{N} \gamma\left(1+\frac{\epsilon_{i}}{4} \pi_{i j}\right) \pi_{i j} A_{i}^{T} P_{j} A_{i}+\Xi_{i} \Gamma \Xi_{i}^{T} \\
< & 0, \forall i \in \mathcal{L} .
\end{aligned}
$$

Thus, a necessary condition for Theorem 2 to hold is given by (28).

Remark 4: It is observed from (28) that when $X_{i} \triangleq \gamma P_{i}>$ $\frac{\mu}{2} I$, the hybrid control strategy $\left(\Pi, K_{i}\right)$ may help us to exponentially stabilize a class of MJLSs which do not satisfy the necessary condition (21) but fulfill (28). This analytically indicates the role of the MDSOF in the proposed hybrid design methodology (see Example 1).

Propositions 3 and 4 establish the relationships between the designed transition probability matrix $\Pi$ and the introduced small scalars $\epsilon_{i}>0$ in the coupled Lyapunov matrix inequalities (5) and the coupled algebraic Riccati matrix inequalities (12).

\section{Estimation of Decay Rate And Decay Coefficient}

To illustrate the exponential mean-square stability of the resultant discrete-time MJLSs, it would be interesting to discuss how to estimate the decay rate $\gamma+\sigma$ and decay coefficient $b$ in Definition 1, where $\gamma$ is a specified lower bound on the decay rate. To this end, the following proposition proposes an approach to estimating the exponential bound for Theorem 1 .

Proposition 5: For a given scalar $\gamma \geq 1$, if Theorem 1 is feasible with $\epsilon_{i}>0$, matrices $X_{i} \triangleq \gamma P_{i}>0$, $\bar{X}_{i} \triangleq\left(\gamma P_{i}\right)^{-1}>0$, and a transition probability matrix $\Pi=\left[\pi_{i j}\right]_{i, j \in \mathcal{L}}$, then an estimated decay rate is

$$
\gamma^{\#} \triangleq \gamma+\sigma=\gamma+\frac{\gamma}{4} \min _{i, j \in \mathcal{L}}\left\{\epsilon_{i} \pi_{i j}\right\},
$$

and a decay coefficient is

$$
b^{\#}=\max _{i \in \mathcal{L}}\left\{\left\|X_{i}\right\|\right\} \cdot \max _{i \in \mathcal{L}}\left\{\left\|\bar{X}_{i}\right\|\right\} .
$$

In this case, the unforced MJLS (1)-(2) is $\gamma$-EMSS with

$$
\mathbf{E}\left\{\|x(k)\|^{2} \mid x_{0}, r_{0}\right\} \leq b^{\#}\left(\gamma^{\#}\right)^{-k}\left\|x_{0}\right\|^{2} .
$$

Proof: As shown in the proof of Theorem 1, inequalities (7)-(8) are equivalent to (9), which can be rewritten as

$$
\begin{aligned}
& -P_{i}+\gamma \sum_{j=1}^{N} \pi_{i j} A_{i}^{T} P_{j} A_{i}+\epsilon_{i} \gamma \sum_{j=1}^{N}\left(\frac{1}{2} \pi_{i j} A_{i}\right)^{T} P_{j}\left(\frac{1}{2} \pi_{i j} A_{i}\right) \\
& =-P_{i}+\sum_{j=1}^{N} \gamma\left(1+\frac{\epsilon_{i}}{4} \pi_{i j}\right) \pi_{i j} A_{i}^{T} P_{j} A_{i}<0
\end{aligned}
$$

By selecting a decay rate as $\gamma^{\#}$ in (30), it follows that (33) implies

$$
-P_{i}+\gamma^{\#} \sum_{j=1}^{N} \pi_{i j} A_{i}^{T} P_{j} A_{i}<0 .
$$


Define a stochastic Lyapunov function candidate as $V(x(k), r(k)=i)=\left(\gamma^{\#}\right)^{k} x^{T}(k) P_{i} x(k)$. Along the solution of the unforced system (1), we have

$$
\begin{aligned}
& \Delta V(x(k), i) \\
= & \mathbf{E}\{V(x(k+1), r(k+1))\}-V(x(k), r(k)=i) \\
= & \left(\gamma^{\#}\right)^{k} x^{T}(k)\left[\gamma^{\#} \sum_{j=1}^{N} \pi_{i j} A_{i}^{T} P_{j} A_{i}-P_{i}\right] x(k) .(35)
\end{aligned}
$$

By combining (34) and (35), one gives

$$
\begin{aligned}
\mathbf{E}\{V(x(k), r(k))\} & \leq V\left(x_{0}, r_{0}\right) \\
& \leq \max _{i \in \mathcal{L}}\left\{\left\|P_{i}\right\|\right\}\left\|x_{0}\right\|^{2} .
\end{aligned}
$$

On the other hand, one also has

$$
\mathbf{E}\{V(x(k), r(k))\} \geq \min _{i \in \mathcal{L}}\left\{\left\|P_{i}\right\|\right\}\left(\gamma^{\#}\right)^{k} \mathbf{E}\left\{\|x(k)\|^{2}\right\} .
$$

By considering $X_{i}=\gamma P_{i}$, we can obtain (32) from (36) and (37) directly.

Next, we will provide a proposition for Theorem 2 to estimate the decay rate. To this end, we first introduce a technical lemma.

Lemma 2: For a scalar $\gamma \geq 1$ and a prescribed scalar $\mu>0$, suppose that the real matrices $X_{i} \triangleq \gamma P_{i}>0$, $\bar{X}_{i} \triangleq\left(\gamma P_{i}\right)^{-1}>0, K_{i}$, and a transition probability matrix $\Pi=\left[\pi_{i j}\right]_{i, j \in \mathcal{L}}$ provide a solution to the conditions in Theorem 2. If for any $i, j \in \mathcal{L}$, there exists an adjustable scalar $\varsigma>0$ such that

$$
\max _{j \in \mathcal{L}}\left\{\pi_{i j}\right\}<\frac{2}{\sqrt{\left(1+\frac{\varsigma \epsilon_{i}^{2}}{16}\right)^{2}+\epsilon_{i}}+1+\frac{\varsigma \epsilon_{i}^{2}}{16}},
$$

then, one has

$$
\begin{aligned}
& \gamma\left(1+\frac{\epsilon_{i}}{4} \pi_{i j}\right)\left(A_{i}+B_{i} K_{i} C_{i}\right)^{T} \pi_{i j} P_{j}\left(A_{i}+B_{i} K_{i} C_{i}\right) \\
& \leq \frac{\pi_{i j}}{\varsigma} \gamma\left(\pi_{i j} A_{i}\right)^{T} P_{j}\left(\pi_{i j} A_{i}\right) \\
& +\gamma\left(1+\frac{\epsilon_{i}}{4} \pi_{i j}\right) A_{i}^{T} \pi_{i j} P_{j} A_{i}+\gamma\left(B_{i} K_{i} C_{i}\right)^{T} P_{j}\left(B_{i} K_{i} C_{i}\right) \\
& +\gamma\left(B_{i} K_{i} C_{i}\right)^{T} P_{j}\left(\pi_{i j} A_{i}\right)+\gamma\left(\pi_{i j} A_{i}\right)^{T} P_{j}\left(B_{i} K_{i} C_{i}\right) .
\end{aligned}
$$

Proof: Suppose the conditions in Theorem 2 are feasible, we define

$$
\begin{aligned}
& J_{1} \triangleq \gamma\left(1+\frac{\epsilon_{i}}{4} \pi_{i j}\right)\left(A_{i}+B_{i} K_{i} C_{i}\right)^{T} \pi_{i j} P_{j}\left(A_{i}+B_{i} K_{i} C_{i}\right), \\
& J_{2} \triangleq \frac{\pi_{i j}}{\varsigma} \gamma\left(\pi_{i j} A_{i}\right)^{T} P_{j}\left(\pi_{i j} A_{i}\right) \\
& +\gamma\left(1+\frac{\epsilon_{i}}{4} \pi_{i j}\right) A_{i}^{T} \pi_{i j} P_{j} A_{i}+\gamma\left(B_{i} K_{i} C_{i}\right)^{T} P_{j}\left(B_{i} K_{i} C_{i}\right) \\
& +\gamma\left(B_{i} K_{i} C_{i}\right)^{T} P_{j}\left(\pi_{i j} A_{i}\right)+\gamma\left(\pi_{i j} A_{i}\right)^{T} P_{j}\left(B_{i} K_{i} C_{i}\right) .
\end{aligned}
$$

Clearly, for $\pi_{i j}=0$, one has $J_{1}=0$ and $J_{2}=$ $\gamma\left(B_{i} K_{i} C_{i}\right)^{T} P_{j}\left(B_{i} K_{i} C_{i}\right)$, which means that the inequality (39) holds. On the other hand, if $\pi_{i j} \neq 0$, we have

$$
J_{1}-J_{2} \leq-\frac{\pi_{i j}}{\varsigma} \gamma\left(\pi_{i j} A_{i}\right)^{T} P_{j}\left(\pi_{i j} A_{i}\right)
$$

$$
\begin{aligned}
& +\gamma \frac{\epsilon_{i}}{4}\left(\pi_{i j} A_{i}\right)^{T} P_{j}\left(\pi_{i j} B_{i} K_{i} C_{i}\right) \\
& +\gamma \frac{\epsilon_{i}}{4}\left(\pi_{i j} B_{i} K_{i} C_{i}\right)^{T} P_{j}\left(\pi_{i j} A_{i}\right) \\
& +\gamma\left(\frac{1}{\pi_{i j}}+\frac{\epsilon_{i}}{4}-\frac{1}{\pi_{i j}^{2}}\right) \\
& \times\left(\pi_{i j} B_{i} K_{i} C_{i}\right)^{T} P_{j}\left(\pi_{i j} B_{i} K_{i} C_{i}\right) \\
& =\gamma \varpi\left(\digamma \otimes P_{j}\right) \varpi^{T},
\end{aligned}
$$

where

$$
\begin{aligned}
& \varpi \triangleq\left[\begin{array}{cc}
\left(\pi_{i j} A_{i}\right)^{T} & \left(\pi_{i j} B_{i} K_{i} C_{i}\right)^{T}
\end{array}\right], \\
& \digamma \triangleq\left[\begin{array}{cc}
-\frac{\pi_{i j}}{\varsigma} & \frac{\epsilon_{i}}{4} \\
\frac{\epsilon_{i}}{4} & \frac{1}{\pi_{i j}}+\frac{\epsilon_{i}}{4}-\frac{1}{\pi_{i j}^{2}}
\end{array}\right] .
\end{aligned}
$$

It is shown from (41) that $\digamma<0$ implies $J_{1} \leq J_{2}$, and $\digamma<0$ if and only if there exists a scalar $\varsigma>0$ such that

$$
-\frac{\pi_{i j}}{\varsigma}<0, \quad \frac{1}{\pi_{i j}}+\frac{\epsilon_{i}}{4}-\frac{1}{\pi_{i j}^{2}}+\frac{\varsigma \epsilon_{i}^{2}}{16 \pi_{i j}}<0 .
$$

It is easy to find that (42) can be guaranteed if

$$
\pi_{i j}<\frac{2}{\sqrt{\left(1+\frac{\varsigma \epsilon_{i}^{2}}{16}\right)^{2}+\epsilon_{i}}+1+\frac{\varsigma \epsilon_{i}^{2}}{16}},
$$

which is further ensured by (38).

As discussed in Problems $1 \mathrm{~b}$ and $2 \mathrm{~b}$ of Section $\mathrm{I}$, the hybrid design involves in the case, $A_{i}+B_{i} K_{i} C_{i} \neq 0$ for any $K_{i} \in \mathbb{R}^{m \times p}$. Based on the above technical lemma, we have the following proposition.

Proposition 6: For a given scalar $\gamma \geq 1$ and a prescribed scalar $\mu>0$, if Theorem 2 is feasible and there exists an adjustable scalar $\varsigma>0$ such that the condition (38) and the following condition holds:

$$
\sum_{j=1}^{N}\left[\mu^{2} \bar{X}_{j}+\left(1-\frac{\pi_{i j}}{\varsigma}\right) \pi_{i j}^{2} X_{j}-2 \mu \pi_{i j} I\right] \geq 0,
$$

then an estimated decay rate is given as

$$
\gamma^{\#} \triangleq \gamma+\sigma=\gamma+\frac{\gamma}{4} \min _{i, j \in \mathcal{L}}\left\{\epsilon_{i} \pi_{i j}\right\}+\min _{i \in \mathcal{L}}\left\{\theta_{i}\right\},
$$

where

$\theta_{i} \triangleq \frac{\lambda_{\min }\left(\sum_{j=1}^{N} A_{i}^{T}\left[\mu^{2} \bar{X}_{j}+\left(1-\frac{\pi_{i j}}{\varsigma}\right) \pi_{i j}^{2} X_{j}-2 \mu \pi_{i j} I\right] A_{i}\right)}{\lambda_{\max }\left(\sum_{j=1}^{N}\left(A_{i}+B_{i} K_{i} C_{i}\right)^{T} \pi_{i j} X_{j}\left(A_{i}+B_{i} K_{i} C_{i}\right)\right)}$,

and a decay coefficient is $b^{\#}$ in (31). In this case, the closedloop MJLS (11) is $\gamma$-EMSS with

$$
\mathbf{E}\left\{\|x(k)\|^{2} \mid x_{0}, r_{0}\right\} \leq b^{\#}\left(\gamma^{\#}\right)^{-k}\left\|x_{0}\right\|^{2} .
$$

Proof: It is noted that (20) can be rewritten as

$$
\begin{aligned}
& -P_{i}+\sum_{j=1}^{N}\left[\mu^{2} A_{i}^{T}\left(\gamma P_{j}\right)^{-1} A_{i}-2 \mu \pi_{i j} A_{i}^{T} A_{i}\right. \\
& +\left(1-\frac{\pi_{i j}}{\varsigma}\right) \gamma\left(\pi_{i j} A_{i}\right)^{T} P_{j}\left(\pi_{i j} A_{i}\right)
\end{aligned}
$$




$$
\begin{aligned}
& +\gamma\left(1+\frac{\epsilon_{i}}{4} \pi_{i j}\right) A_{i}^{T} \pi_{i j} P_{j} A_{i}+\frac{\pi_{i j}}{\varsigma} \gamma\left(\pi_{i j} A_{i}\right)^{T} P_{j}\left(\pi_{i j} A_{i}\right) \\
& +\gamma\left(B_{i} K_{i} C_{i}\right)^{T} P_{j}\left(\pi_{i j} A_{i}\right)+\gamma\left(\pi_{i j} A_{i}\right)^{T} P_{j}\left(B_{i} K_{i} C_{i}\right) \\
& \left.+\gamma\left(B_{i} K_{i} C_{i}\right)^{T} P_{j}\left(B_{i} K_{i} C_{i}\right)\right]<0,
\end{aligned}
$$

which is also equivalent to (14)-(15).

According to Lemma 2, if (38) is true, the inequality (48) can guarantee the following inequality:

$$
\begin{aligned}
& -P_{i}+\sum_{j=1}^{N}\left[\mu^{2} A_{i}^{T}\left(\gamma P_{j}\right)^{-1} A_{i}-2 \mu \pi_{i j} A_{i}^{T} A_{i}\right. \\
& \left.+\left(1-\frac{\pi_{i j}}{\varsigma}\right) \gamma\left(\pi_{i j} A_{i}\right)^{T} P_{j}\left(\pi_{i j} A_{i}\right)\right] \\
& +\sum_{j=1}^{N} \gamma\left(1+\frac{\epsilon_{i}}{4} \pi_{i j}\right)\left(A_{i}+B_{i} K_{i} C_{i}\right)^{T} \pi_{i j} P_{j}\left(A_{i}+B_{i} K_{i} C_{i}\right) \\
& <0 .
\end{aligned}
$$

Notice that if condition (44) is also feasible for the specified scalar $\varsigma>0$, there must exist a scalar $\theta_{i} \geq 0$ in (46) satisfying

$$
\begin{aligned}
& \theta_{i} \sum_{j=1}^{N}\left(A_{i}+B_{i} K_{i} C_{i}\right)^{T} \pi_{i j} X_{j}\left(A_{i}+B_{i} K_{i} C_{i}\right) \\
& \leq \sum_{j=1}^{N} A_{i}^{T}\left[\mu^{2} \bar{X}_{j}+\left(1-\frac{\pi_{i j}}{\varsigma}\right) \pi_{i j}^{2} X_{j}-2 \mu \pi_{i j} I\right] A_{i} .
\end{aligned}
$$

Select an decay rate as $\gamma^{\#}$ in (45) and define the Lyapunov functional as $V(x(k), r(k)=i)=\left(\gamma^{\#}\right)^{k} x^{T}(k) P_{i} x(k)$ for the closed-loop system (11). Then, by a similar line of arguments as in the proof of Proposition 5, it can be shown that exponential mean-square stability condition (47) also holds for the closed-loop system (11).

Remark 5: The inequality in (19) implies that there must exist a matrix $\Delta_{i} \in \mathbb{R}^{n \times n}, \Delta_{i}>0, \forall i \in \mathcal{L}$, satisfying

$$
\begin{aligned}
& -\sum_{j=1}^{N}\left(\pi_{i j} A_{i}\right)^{T}\left(\gamma P_{j}\right)\left(\pi_{i j} A_{i}\right)+\Delta_{i} \\
\leq & \mu^{2} \sum_{j=1}^{N} A_{i}^{T}\left(\gamma P_{j}\right)^{-1} A_{i}-2 \mu A_{i}^{T} A_{i}
\end{aligned}
$$

where $\Delta_{i}$ is chosen as the following form:

$$
\begin{aligned}
\Delta_{i} \triangleq & \theta_{i} \sum_{j=1}^{N}\left(A_{i}+B_{i} K_{i} C_{i}\right)^{T} \pi_{i j} X_{j}\left(A_{i}+B_{i} K_{i} C_{i}\right) \\
& +\sum_{j=1}^{N} A_{i}^{T} \frac{\pi_{i j}^{3}}{\varsigma} X_{j} A_{i}
\end{aligned}
$$

with some sufficiently small $\theta_{i} \geq 0$ and sufficiently large $\varsigma>$ 0 . It is clear that the term, $\min _{i \in \mathcal{L}}\left\{\theta_{i}\right\}$, in (45) is motivated by $\Delta_{i}$ in (52). This means that although some conservatism may be yielded due to utilizing inequality (19), it also brings room for improvement in the estimation of the decay rate of the hybrid controlled MJLSs as shown in Proposition 6.

Remark 6: It is observed from Proposition 6 that the estimation approach on the decay rate of the hybrid design depends on the conditions (38) and (44) associated with an adjustable scalar $\varsigma>0$. Specifically, with the increase of $\varsigma$, condition (38) will be difficultly ensured, but condition (44) may hold more easily, vice versa. These two conditions construct a game theoretic problem for Theorem 2, which can be solved by linear search of $\varsigma$ for some obtained solutions of Theorem 2 such that conditions (38) and (44) hold simultaneously. Notice that $\epsilon_{i}$ is a scalar parameter which must be kept sufficiently small [18], [19] and inequality (19) holds for any $P_{i}>0$, thus the estimation approach in Proposition 6 can be applied in almost all feasible solutions of Theorem 2. In the case that Proposition 6 does not work, the estimated decay rate can at least be given as $\gamma$ according to Theorem 2 .

Remark 7: While Proposition 6 is applicable for an obtained solution, the feasible range $\left[\varsigma_{\min }, \varsigma_{\max }\right]$ of $\varsigma$, which ensures conditions (38) and (44) simultaneously, can be determined directly by a simple linear searching for a chosen small step $\tau>0$. Furthermore, it is easy to find from (45) that over the feasible range $\left[\varsigma_{\min }, \varsigma_{\max }\right]$, the maximum estimation decay rate in Proposition 6 can be achieved by choosing $\varsigma=\varsigma_{\max }$.

Propositions 5 and 6 can provide an estimate on the decay rate of the discrete-time MJLSs. Indeed, for a given scalar $\gamma \geq 1$, it has been proven that the actual decay rate $\bar{\gamma}$ will not be smaller than $\gamma^{\#}$. That is,

$$
\bar{\gamma} \geq \gamma^{\#} \geq \gamma
$$

\section{E. Performance Enhancement}

To better apply the above results in practical application, two kinds of optimization problems may be considered: (i) It can be concluded from (3) that a larger $\sum_{i=1}^{N} \pi_{i i}$ implies less stochastic switchings in probability. For some practical applications, for instance, when designing a stabilizing transition probability matrix, a lower switching frequency always means lower energy requirements. Therefore, it is very meaningful to maximize the obtained value of $\sum_{i=1}^{N} \pi_{i i}$ in hybrid design. (ii) It is well known that a larger decay rate implies a faster convergence of the system trajectories. To this end, the maximum decay rate achievable may be maximized in such applications.

Based on the above discussions, the 1st kind of optimization problems is formulated as follows based on Theorems 1 and 2:

$$
\begin{aligned}
& \max _{\epsilon_{i}, \mu} \sum_{i=1}^{N} \pi_{i i} \\
& \text { s.t. }(7)-(8) \text { or }(14)-(15) .
\end{aligned}
$$

The 2nd kind of optimization problem for Proposition 5 is considered as

$$
\begin{aligned}
& \max _{\epsilon_{i}} \nu_{i j} \triangleq \min _{i, j \in \mathcal{L}}\left\{\epsilon_{i} \pi_{i j}\right\} \\
& \text { s.t. }(7)-(8),
\end{aligned}
$$

and the one for Proposition 6 is formulated as

$$
\begin{aligned}
& \max _{\epsilon_{i}, \mu, \varsigma} \nu_{i j} \triangleq \frac{\gamma}{4} \min _{i, j \in \mathcal{L}}\left\{\epsilon_{i} \pi_{i j}\right\}+\min _{i \in \mathcal{L}}\left\{\theta_{i}\right\} \\
& \text { s.t. (14) }-(15) \text { with (38) and (44), }
\end{aligned}
$$


along with the decay coefficient $b^{\#}$ obtained from (31).

Remark 8: It is worth mentioning that the above two kinds of optimization problems can be used in conjunction with each other according to the actual situation of the practical applications. For example, one may want to first solve optimization problem (54) with the non-uniqueness of the solution $\left\{\epsilon_{i}\right\}$ for optimizing the problem (55). This procedure can be carried out iteratively so that a slow switching and fast decay rate design may be achieved.

\section{Hybrid Design With Multiple Constraints}

Note that the significance of the conditions in Theorem 2 lies in that there is no any coupling relationship between matrices $P_{i}$, controller gains $K_{i}$, and the transition probabilities $\pi_{i j}$. This feature brings two advantages to the proposed hybrid control approach. One is that the hybrid design is noniterative, that is, we can solve all the parameters, including the MDSOF controllers, directly using nonconvex optimization. The other is that it allows us to deal with some additional constraints for practical hybrid design without imposing more requirements on $P_{i}$. Based on these two advantages, this section is devoted to discussing how to overcome two classes of possible constraints on the practical hybrid design.

1) Gain constraints: In many practical applications, the magnitude constraints on controller gains should be considered [21], [22]. For the MDSOF controller gains $K_{i}$ in (6), we consider the following gain constraint conditions:

$$
K_{i}^{T} K_{i} \leq \delta_{K_{i}}^{2} I, i \in \mathcal{L},
$$

where $\delta_{K_{i}}>0, i \in \mathcal{L}$, are the specified upper bounds on $\left\|K_{i}\right\|$.

For this case, the hybrid design of MDSOF control can be obtained by solving the conditions in Theorem 2 with the following LMIs:

$$
\left[\begin{array}{cc}
-\delta_{K_{i}}^{2} I & K_{i}^{T} \\
K_{i} & -I
\end{array}\right] \leq 0, i \in \mathcal{L}
$$

2) Structured transition probability matrix: In practical application, the transition probability matrix may be imposed on various structured constraints. For instance, the Gossip Markov Chain can be mapped onto a NoC as in Fig. 1 where the Markov transition probability matrix is given as

$$
\begin{aligned}
& \Pi_{G}= \\
& {\left[\begin{array}{cccc}
\alpha & \beta & 1-(\alpha+\beta) & 0 \\
1-(\alpha+\beta) & \alpha & 0 & \beta \\
\beta & 0 & \alpha & 1-(\alpha+\beta) \\
0 & 1-(\alpha+\beta) & \beta & \alpha
\end{array}\right]}
\end{aligned}
$$

where $\alpha \in[0,1]$ and $\beta \in[0,1]$ are the adjustable parameters in NoC [12], [17]. It can be observed that some elements in the transition probability matrix are fixed a priori, and it is highly structured. Nevertheless, Theorems 1 and 2 in this work can incorporate these constraints directly (see Example 3).

Another notable structural constraints on the transition probability matrix is that some transition probabilities may be

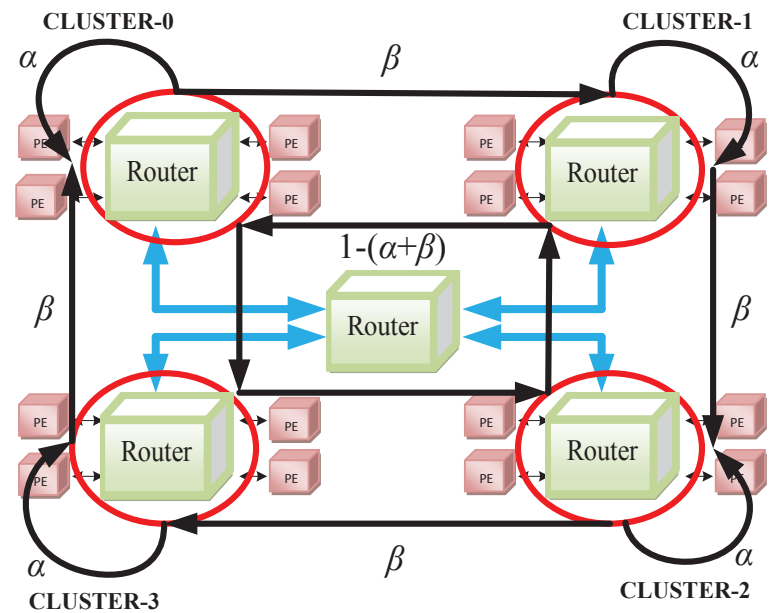

Fig. 1. A NoC mapped with Gossip Markov Chain [12] ("PE" is the abbreviation of "processing element")

imposed by the specified interval constraints [23]-[25] subject to

$$
\underline{\pi}_{i j} \leq \pi_{i j} \leq \bar{\pi}_{i j}, i, j \in \mathcal{L}
$$

where $\underline{\pi}_{i j} \geq 0$ and $\bar{\pi}_{i j} \leq 1$ are given scalars. These LMIs can be easily incorporated into Theorems 1 and 2 .

Remark 9: The MDSOF control of discrete-time MJLSs has gained research interest as seen in [26]-[29]. It should be remarked that the major obstacle in MDSOF synthesis of MJLSs is not only the nonconvexity of the SOF problem itself, but also a coupled LMI condition for the stochastic stability of MJLSs, which poses a severe problem in controller synthesis [27]. Clearly, if a completely structured transition probability matrix $\Pi$ is specified in Theorem 2 , that is, all its elements are given, the proposed hybrid design approach can be applied to solve the MDSOF control problems as in [26]-[29] directly, but not vice versa. This shows a key advantage of our approach compared with the existing works on MDSOF synthesis with fixed transition probability matrix.

Remark 10: Since there is no coupling relationship between the decision variables, the results obtained in this work can be readily extended to the polytopic uncertain case by utilizing similar techniques employed in [30], [31].

\section{ILluStrative EXAMPLES}

To overcome the difficulties in choosing a set of modedependent parameters $\epsilon_{i}>0$, the gridding technique in [32] is utilized in this work to solve the obtained conditions. To this end, we set

$$
\epsilon_{i}=\frac{\kappa_{i}}{1-\kappa_{i}}, \forall i \in \mathcal{L} .
$$

It is clear from (61) that one may have $\epsilon_{i} \in(0,+\infty)$ if and only if the scalars $\kappa_{i} \in(0,1)$. Then, for any mode $i \in \mathcal{L}$, we select a set of grid points as follows:

$$
\kappa_{i}^{h}=h d_{i}, h=1,2, \ldots, \zeta_{i},
$$

where $d_{i}>0$ is a small fixed step size, and $\zeta_{i}=\left\lceil\frac{1}{d_{i}}\right\rceil-1$. For each grid point, one can solve the proposed LMIs with a 
rank constraint inequality by means of Newton-like algorithm as in [33].

In the following examples, we use the default settings in LMIRank [34] running on the MATLAB 2010a platform. It is worth pointing out that the solution is initial value-dependent. In LMIRank, the initial value is generated and optimized via solving a default trace heuristic algorithm. However, LMIRank cannot guarantee finding a solution in any specified feasible point $\left(\kappa_{i}^{1}, \kappa_{i}^{2}, \ldots, \kappa_{i}^{h}\right)$ and thus the obtained feasible area may be incomplete in certain regions within the parameter space. Nevertheless, according to the authors' numerical experience, LMIRank can be quite effective in yielding good results in most cases.

In the sequel, three examples are provided to show the effectiveness of the proposed approaches.

\section{A. Example 1}

This part gives a design example for Problem 1 with solving the optimization problem (54). Consider two discrete-time linear subsystems with system matrices as

$$
A_{1}=\left[\begin{array}{cc}
-1.2 & 0 \\
0 & 0.7
\end{array}\right], A_{2}=\left[\begin{array}{cc}
0.8 & 0 \\
-0.1 & -1.1
\end{array}\right] .
$$

It is easy to find that neither $A_{1}$ nor $A_{2}$ is asymptotically stable. Our aim is to design a hybrid control strategy such that the constructed discrete-time MJLS is $\gamma$-EMSS with the decay rate larger than $\gamma=1.01$. The fixed step sizes in (61) are given as $d_{1}=d_{2}=0.01$, that is, overall $99 \times 99$ points are checked for both Theorems 1 and 2 .

- Problem 1a. Utilizing Theorem 1, we have found 1454 feasible solutions for this example as depicted in Fig. 2. Consider the optimization problem in (54), the maximum value of $\sum_{i=1}^{2} \pi_{i i}=1.2450$ is found at $\left(\kappa_{1}, \kappa_{2}\right)=(0.04,0.03)$ where

$$
\begin{aligned}
& \Pi=\left[\begin{array}{ll}
0.5195 & 0.4805 \\
0.2745 & 0.7255
\end{array}\right], \\
& X_{1}=\left[\begin{array}{cc}
0.8273 & -0.0187 \\
-0.0187 & 0.3692
\end{array}\right], \bar{X}_{1}=\left[\begin{array}{cc}
1.2102 & 0.0614 \\
0.0614 & 2.7118
\end{array}\right], \\
& X_{2}=\left[\begin{array}{ll}
0.2831 & 0.0659 \\
0.0659 & 1.1454
\end{array}\right], \bar{X}_{2}=\left[\begin{array}{cc}
3.5800 & -0.2061 \\
-0.2061 & 0.8849
\end{array}\right] .
\end{aligned}
$$

By Proposition 5, an estimated decay rate is given as $\gamma^{\#}=$ 1.0121 with the corresponding decay coefficient $b^{\#}=4.1367$. Utilizing 10000 independent simulation trials with the initial condition $x(0)=\left[\begin{array}{ll}1 & -2\end{array}\right]^{T}$, the averaged trajectory of $\|x(k)\|^{2}$ and the exponential bound are shown in Fig. 3, from which one can observe that $\mathbf{E}\left\{\|x(k)\|^{2}\right\} \leq 4.1367 \times$ $1.0121^{-k}\|x(0)\|^{2}$.

- Problem 1b. Suppose that the input and output matrices for the above two linear subsystems are

$B_{1}=\left[\begin{array}{l}0 \\ 2\end{array}\right], C_{1}=\left[\begin{array}{ll}0 & 1\end{array}\right], B_{2}=\left[\begin{array}{l}1 \\ 0\end{array}\right], C_{2}=\left[\begin{array}{ll}1 & 0\end{array}\right]$.

It is easily checked that neither $\left\{A_{1}, B_{1}, C_{1}\right\}$ nor $\left\{A_{2}, B_{2}, C_{2}\right\}$ is output controllable for SOF controller. By Theorem 2 with setting $\mu=1$, we find 3533 feasible solutions for this problem as shown in Fig. 4. It is found

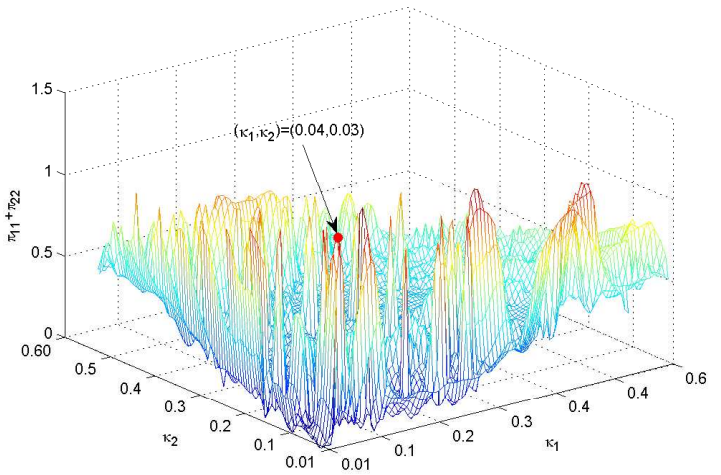

Fig. 2. $\quad \sum_{i=1}^{2} \pi_{i i}$ vs. $\left(\kappa_{1}, \kappa_{2}\right)$ for Problem 1a

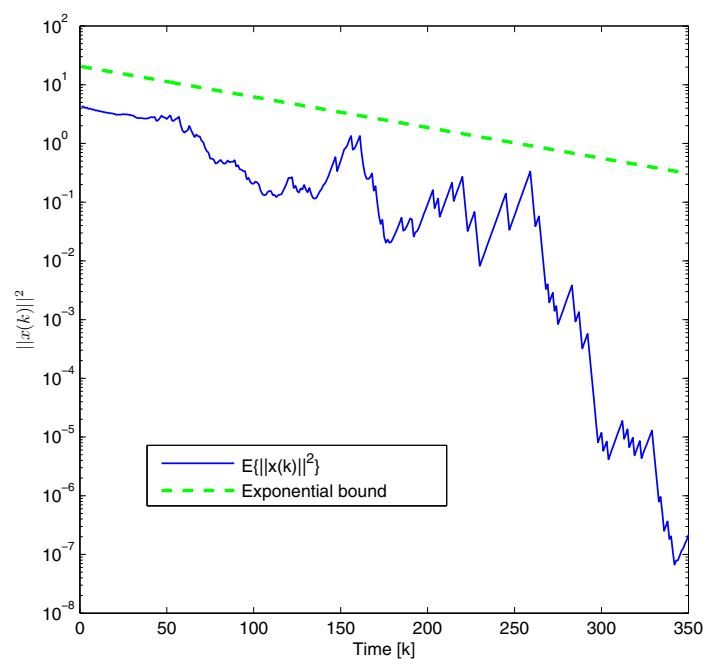

Fig. 3. $\quad \mathbf{E}\left\{\|x(k)\|^{2}\right\}$ and exponential bound (Problem 1a)

that the maximum value of $\sum_{i=1}^{2} \pi_{i i}=0.9076$ is achieved at $\left(\kappa_{1}, \kappa_{2}\right)=(0.20,0.24)$ with

$\Pi=\left[\begin{array}{ll}0.3758 & 0.6242 \\ 0.4682 & 0.5318\end{array}\right], K_{1}=-0.2050, K_{2}=-0.3968$,
$X_{1}=\left[\begin{array}{cc}2.7538 & -0.1346 \\ -0.1346 & 1.3845\end{array}\right], \bar{X}_{1}=\left[\begin{array}{cc}0.3649 & 0.0355 \\ 0.0355 & 0.7257\end{array}\right]$,
$X_{2}=\left[\begin{array}{ll}0.8986 & 0.3695 \\ 0.3695 & 5.0362\end{array}\right], \bar{X}_{2}=\left[\begin{array}{cc}1.1475 & -0.0842 \\ -0.0842 & 0.2047\end{array}\right]$.

One can verify that the above matrices satisfy the conditions in (38) and (44). According to Remark 7, the feasible range of $\varsigma$ is searched over $[1.5,134.6]$ for a chosen small step $\tau=0.1$. Thus, let $\varsigma=134.6$ in Proposition 6, we estimated the maximum allowable decay rate $\gamma^{\#}=1.1169$ and the corresponding decay coefficient $b^{\#}=5.8544$. With the initial condition $x(0)=\left[\begin{array}{ll}-1 & 1\end{array}\right]^{T}$, the averaged trajectory of $\|x(k)\|^{2}$ over 10000 independent simulation trials and its estimated exponential bound are plotted in Fig. 5. It can be seen that the hybrid design $\left(\Pi, K_{i}\right)$ can exponentially stabilize the MJLS as $\mathbf{E}\left\{\|x(k)\|^{2}\right\} \leq 5.8544 \times 1.1169^{-k}\|x(0)\|^{2}$, but not $\Pi$ alone, which also demonstrates the analysis in Remark 
4.

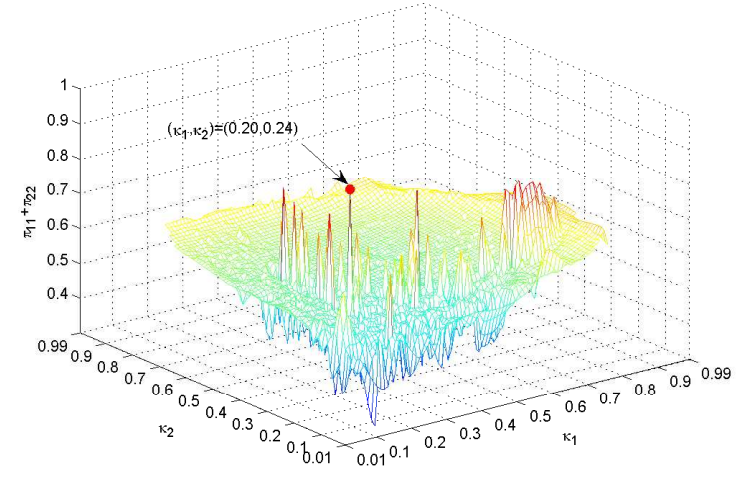

Fig. 4. $\sum_{i=1}^{2} \pi_{i i}$ vs. $\left(\kappa_{1}, \kappa_{2}\right)$ for Problem $1 \mathrm{~b}$.

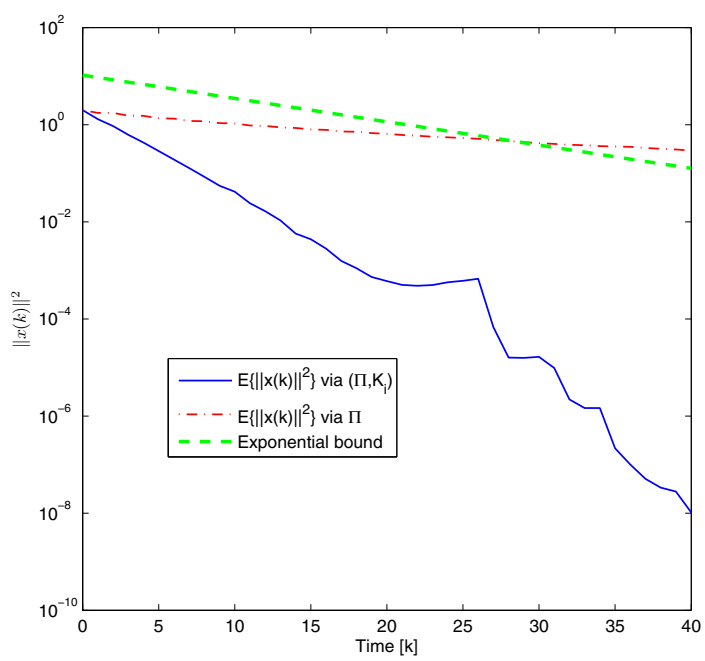

Fig. 5. $\quad \mathbf{E}\left\{\|x(k)\|^{2}\right\}$ and exponential bound (Problem 1b)

\section{B. Example 2}

A numerical example for Problem 2 in considered based on optimization problem (55). Consider two discrete-time linear subsystems as follows:

$$
A_{1}=\left[\begin{array}{ccc}
0.8 & 0 & 0 \\
0 & -0.9 & 0 \\
0 & 0 & -0.9
\end{array}\right], A_{2}=\left[\begin{array}{ccc}
-1 & 0.1 & 0 \\
0 & 0 & 0.2 \\
0 & 0 & 0.1
\end{array}\right]
$$

It is clear that $A_{1}$ is asymptotically stable but not $A_{2}$. For $\gamma=1.2$, similar to Example 1, the fixed step sizes in (61) are chosen as $d_{1}=d_{2}=0.01$.

-Problem 2a. Theorem 1 finds 4865 feasible solutions in this case. Now, we consider the optimization problem in (55). The maximum value of $\min _{i, j \in \mathcal{L}}\left\{\epsilon_{i} \pi_{i j}\right\}=0.3376$ is found to be $\left(\kappa_{1}, \kappa_{2}\right)=(0.58,0.62)$ as shown in Fig. 6 , where

$$
\Pi=\left[\begin{array}{ll}
0.7555 & 0.2445 \\
0.7272 & 0.2728
\end{array}\right],
$$

$$
\begin{aligned}
& X_{1}=\left[\begin{array}{ccc}
7.2573 & 0.1907 & -0.0192 \\
0.1907 & 9.4710 & -1.6949 \\
-0.0192 & -1.6949 & 2.6897
\end{array}\right], \\
& \bar{X}_{1}=\left[\begin{array}{ccc}
0.1372 & -0.0027 & -0.0009 \\
-0.0027 & 0.1186 & 0.0750 \\
-0.0009 & 0.0750 & 0.4188
\end{array}\right], \\
& X_{2}=\left[\begin{array}{ccc}
10.2032 & -1.0413 & 0.0461 \\
-1.0413 & 0.4648 & 0.0411 \\
0.0461 & 0.0411 & 0.6505
\end{array}\right], \\
& \bar{X}_{2}=\left[\begin{array}{ccc}
0.1274 & 0.2877 & -0.0273 \\
0.2877 & 2.8109 & -0.1980 \\
-0.0273 & -0.1980 & 1.5502
\end{array}\right] .
\end{aligned}
$$

According to (55), an estimated maximum decay rate is $\gamma^{\#}=1.3013$ with the decay coefficient $b^{\#}=29.6163$. By utilizing 10000 independent simulation trials with the initial condition $x(0)=\left[\begin{array}{ccc}-3 & 1 & 2\end{array}\right]^{T}$, the averaged trajectory of $\|x(k)\|^{2}$ and the corresponding exponential bound are depicted in Fig. 7, from which one can observe that $\mathbf{E}\left\{\|x(k)\|^{2}\right\} \leq$ $29.6163 \times 1.3013^{-k}\|x(0)\|^{2}$ under the designed transition probability matrix $\Pi$. Besides, one can also see from Fig. 7 that the designed transition probability matrix $\Pi$ yields quicker convergence and smaller bound for $\mathbf{E}\left\{\|x(k)\|^{2}\right\}$ than a fixed transition probability matrix $\Pi_{0} \triangleq\left[\begin{array}{lll}1 & 0 ; 1 & 0\end{array}\right]$, which has further confirmed the advantage of the proposed transition probability synthesis approach.

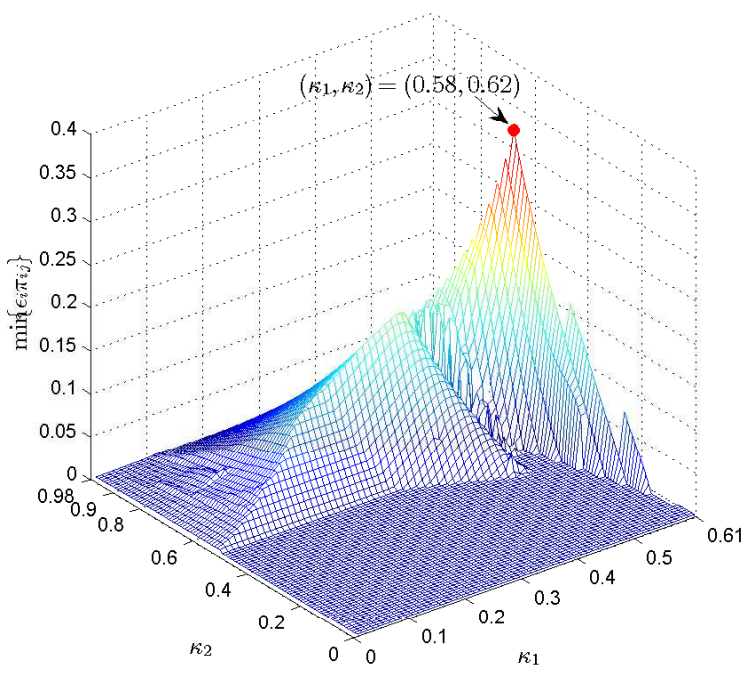

Fig. 6. $\min _{i, j \in \mathcal{L}}\left\{\epsilon_{i} \pi_{i j}\right\}$ vs. $\left(\kappa_{1}, \kappa_{2}\right)$ for Problem 2a

-Problem 2b. Suppose that the input and output matrices for the above two subsystems are

$B_{1}=B_{2}=\left[\begin{array}{l}0 \\ 1 \\ 1\end{array}\right], C_{1}=\left[\begin{array}{lll}0 & 0 & 0\end{array}\right], C_{2}=\left[\begin{array}{lll}0 & 1 & 1\end{array}\right]$.

It is easily shown that neither $\left\{A_{1}, B_{1}, C_{1}\right\}$ nor $\left\{A_{2}, B_{2}, C_{2}\right\}$ is output controllable for SOF controller. Let $K_{1}=0$. By Theorem 2 with choosing $\mu=0.5$, we find 2475 


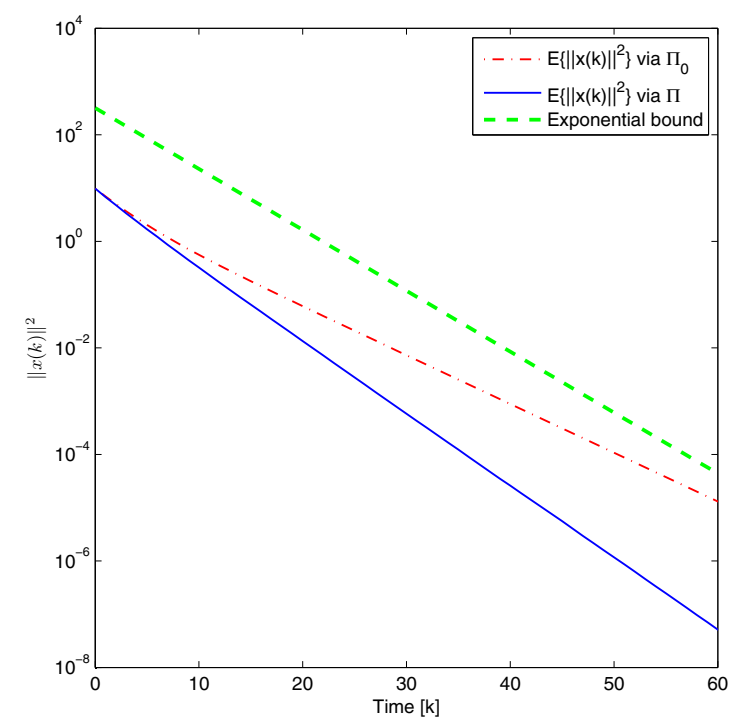

Fig. 7. $\quad \mathbf{E}\left\{\|x(k)\|^{2}\right\}$ and exponential bound (Problem 2a)

feasible solutions. Choose the step size $\tau$ in Remark 7 as 0.1 , we then examine all feasible solutions by Proposition 6, and estimated the maximum allowable decay rate is searched as $\gamma^{\#}=1.2437$ at $\left(\kappa_{1}, \kappa_{2}\right)=(0.30,0.30)$ for $\varsigma=38.6$ with

$$
\begin{aligned}
& \Pi=\left[\begin{array}{ll}
0.5470 & 0.4530 \\
0.6603 & 0.3397
\end{array}\right], K_{2}=0.0125, \\
& X_{1}=\left[\begin{array}{lll}
0.8108 & 0.0097 & 0.0001 \\
0.0097 & 1.0379 & 0.0000 \\
0.0001 & 0.0000 & 1.0359
\end{array}\right], \\
& \bar{X}_{1}=\left[\begin{array}{ccc}
1.2335 & -0.0115 & -0.0001 \\
-0.0115 & 0.9635 & -0.0000 \\
-0.0001 & -0.0000 & 0.9654
\end{array}\right], \\
& X_{2}=\left[\begin{array}{ccc}
1.2204 & -0.0360 & -0.0003 \\
-0.0360 & 0.9641 & 0.0000 \\
-0.0003 & 0.0000 & 0.9630
\end{array}\right], \\
& \bar{X}_{2}=\left[\begin{array}{ccc}
0.8203 & 0.0307 & 0.0003 \\
0.0307 & 1.0384 & -0.0000 \\
0.0003 & -0.0000 & 1.0385
\end{array}\right],
\end{aligned}
$$

along with the corresponding decay coefficient $b^{\#}=1.5122$.

With the initial condition $x(0)=\left[\begin{array}{lll}1 & 3 & -2\end{array}\right]^{T}$, the averaged trajectory of $\|x(k)\|^{2}$ for 10000 independent simulation samples and the corresponding exponential bound are depicted in Fig. 8, which shows the hybrid design can exponentially mean-square stabilize the discrete-time MJLSs with $\mathbf{E}\left\{\|x(k)\|^{2}\right\} \leq 1.5122 \times 1.2437^{-k}\|x(0)\|^{2}$. In addition, by comparing with a fixed transition probability matrix $\Pi_{0} \triangleq$ [1 $0 ; 10]$ with any gain $K_{20}$, the superiority of the synthesized hybrid control strategy $\left(\Pi, K_{2}\right)$ has been illustrated in Fig. 8 as well.

It is worth pointing out that for a given lower bound $\gamma$ on the decay rate, Proposition 6 ensures that the decay rate of the hybrid controlled MJLSs must be larger than or equal to the maximum estimated value $\gamma^{\#}$. However, this also means that, for a certain feasible solution, there may exist a possibility of a higher decay rate for the controlled system in magnitude. Fixing the matrices $K_{1}, K_{2}, P_{1}=\gamma^{-1} X_{1}$, $P_{2}=\gamma^{-1} X_{2}$ and the transition probabilities $\pi_{i j}$ in (12), we now increase $\gamma$ incrementally until (12) is no longer feasible. Then, a maximum allowable decay rate $\gamma$ is found as 1.2714 , which corresponds to a $2.18 \%$ difference from the maximum estimated decay rate $\gamma^{\#}$ using Proposition 6. Even though this result may reflect some conservatism in the estimation approach given in Proposition 6, it still illustrates that the actual decay rate of the hybrid controlled MJLSs is at least no less than $\gamma^{\# \text {. }}$

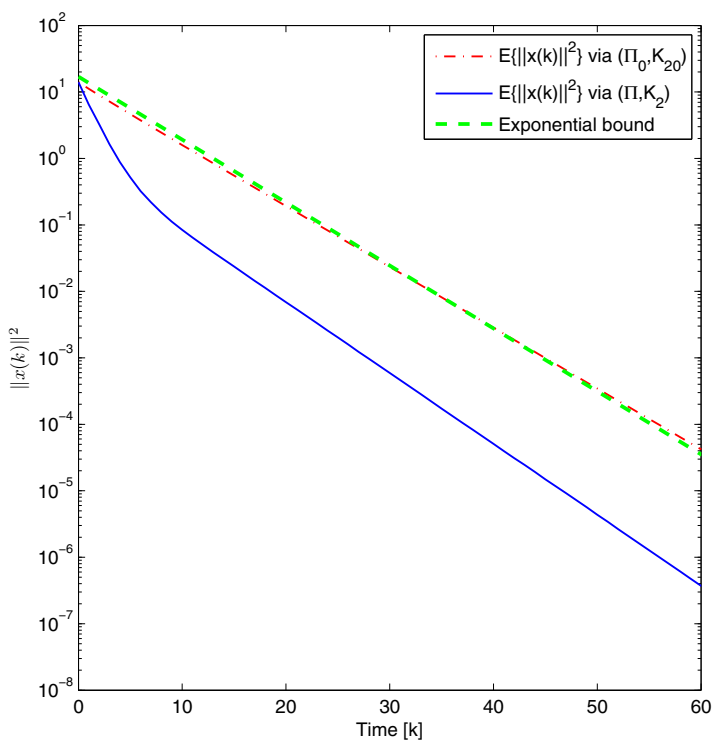

Fig. 8. $\quad \mathbf{E}\left\{\|x(k)\|^{2}\right\}$ and exponential bound (Problem 2b)

\section{Example 3}

The objective of this example is to design a Gossip Markov transition probability matrix $\Pi_{G}$ in (59) to exponentially mean-square stabilize the following four discrete-time linear subsystems:

$$
\begin{aligned}
& A_{1}=\left[\begin{array}{cc}
-1.1 & 0 \\
0 & 0.7
\end{array}\right], A_{2}=\left[\begin{array}{cc}
-0.5 & 0 \\
0 & 1.1
\end{array}\right], \\
& A_{3}=\left[\begin{array}{cc}
1.2 & 0 \\
0 & 0.4
\end{array}\right], A_{4}=\left[\begin{array}{cc}
-1.1 & 0 \\
0 & -0.5
\end{array}\right] .
\end{aligned}
$$

Clearly, none of them is stable. In particular, suppose that the decay rate is required to be larger than $\gamma=1.1$, and a transition probability constraint in (60) with $0.15 \leq \alpha \leq$ 1. By employing Theorem 1 with $d_{1}=d_{2}=d_{3}=d_{4}=$ 0.03 in (61), a feasible solution is found at $\left(\kappa_{1}, \kappa_{2}, \kappa_{3}, \kappa_{4}\right)=$ $(0.09,0.09,0.12,0.21)$ as

$$
\alpha=0.2621, \beta=0.7770 .
$$

With $x(0)=\left[\begin{array}{ll}-1 & 2\end{array}\right]^{T}$, the trajectories of the two components of $x(k)$ are shown in Fig. 9. This example shows 
the potential of the proposed design methodology in practical applications with transition probability matrix constrained on both structure and magnitude.

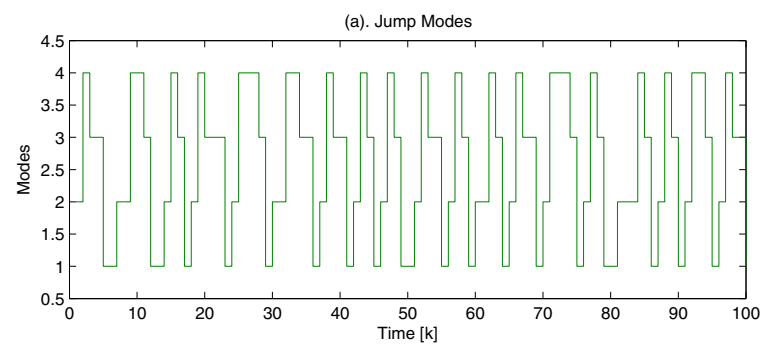

(b). Evolution of State

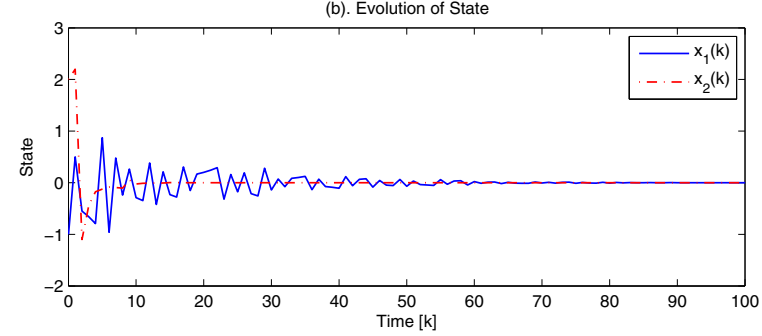

Fig. 9. Stabilization via the designed Gossip Markov probability matrix $\Pi_{G}$

\section{CONCLUSIONS}

This work has investigated the hybrid design problem of transition probability matrix and static output-feedback control so as to achieve the exponential stability of discretetime MJLSs. By the mode-dependent parametric approach, a necessary and sufficient condition for designing the stabilizing transition probability matrix has been established. Moreover, sufficient conditions for MDSOF control with designing transition probability matrix are attained. Besides, several fundamental and interesting problems have been also discussed and illustrated by examples. Under the proposed hybrid design framework, the following two directions may be considered in the future: (1) the multi-performance-guaranteed hybrid design problems to discrete-time MJLSs, such as $H_{\infty}$ hybrid design and mixed $H_{2} / H_{\infty}$ hybrid design; and (2) the hybrid design approaches for various discrete-time MJLSs, such as singular MJLSs, MJLSs with delays and stochastic MJLSs.

\section{ACKNOWLEDGMENT}

The authors would like to thank the Associate Editor and anonymous reviewers for their constructive comments based on which the quality of the work has been improved.

\section{REFERENCES}

[1] O. L. V. Costa, M. D. Fragoso, and R. P. Marques, Discrete-Time Markov Jump Linear Systems, London: Springer-Verlag, 2005.

[2] B. Chen, Y. Niu, and Y. Zou, "Adaptive sliding mode control for stochastic Markovian jumping systems with actuator degradation", Automatica, vol. 49, no. 6, pp. 1748-1754, 2013.

[3] P. Shi, M. Liu, and L. Zhang, "Fault-tolerant sliding-mode-observer synthesis of Markovian jump systems using quantized measurements", IEEE Transactions on Industrial Electronics, vol. 62, no. 9, pp. 5910 5918, 2015.
[4] Z. Wu, H. Su, and J. Chu, "Output feedback stabilization for discrete singular systems with random abrupt changes", International Journal of Robust and Nonlinear Control, vol. 20, no. 17, pp. 1945-1957, 2010.

[5] L. Zhang, and J. Lam, "Necessary and sufficient conditions for analysis and synthesize of Markov jump linear systems with incomplete transition descriptions", IEEE Transactions on Automatic Control, vol. 55, no. 7, pp. 1695-1701, 2010.

[6] J. C. Geromel, A. P. C. Gonçalves, and A. R. Fioravanti, "Dynamic output feedback control of discrete-time Markov jump linear systems through linear matrix inequalities", SIAM Journal on Control and Optimization, vol. 48, no. 2, pp. 573-593, 2009.

[7] P. Bolzern, P. Colaneri, and G. De Nicolao, "Almost sure stability of Markov jump linear systems with deterministic switching", IEEE Transactions on Automatic Control, vol. 58, no. 1, pp. 209-214, 2013.

[8] D. Liberzon, and A. S. Morse, "Basic problems in stability and design of switched systems", IEEE Control Systems Magazine, vol. 19, no. 5, pp. 59-70, 1999.

[9] J. Feng, J. Lam, and Z. Shu, "Stabilization of Markovian systems via probability rate synthesis and output feedback", IEEE Transactions on Automatic Control, vol. 55, no. 3, pp. 773-777, 2010.

[10] S. Ma, and J. Xiong, "Stabilization of time-delay Markovian jump systems via probability rate synthesis and state feedback", IFAC Proceedings Volumes, vol. 47, no. 3, pp. 10415-10420, 2014.

[11] P. Zhang, J. Cao, and G. Wang, "Mode-independent guaranteed cost control of singular Markovian delay jump systems with switching probability rate design", International Journal of Innovative Computing, Information and Control, vol. 10, no. 4, pp. 1291-1303, 2014.

[12] V. K. Sehgal, "Markovian models based stochastic communication in networks-in-package", IEEE Transactions on Parallel and Distrbuted Systems, vol. 26, no. 10, pp. 2806-2821, 2015.

[13] J. Feng, J. Lam, S. Xu, and Z. Shu, "Stabilisation of stochastic systems with optimal decay rate", IET Control Theory and Applications, vol. 2, no. 1, pp. 1-6, 2008.

[14] Z. Wang, Y. Liu, and X. Liu, "Exponential stabilization of a class of stochastic system with Markovian jump parameters and mode-dependent mixed time-delays", IEEE Transactions on Automatic Control, vol. 55, no. 7, pp. 1656-1662, 2010.

[15] Z. Shu, J. Lam, and J. Xiong, "Non-fragile exponential stability assignment of discrete-time linear systems with missing data in actuators", IEEE Transactions on Automatic Control, vol. 54, no. 3, pp. 625-630, 2009.

[16] Z. Shu, J. Xiong, and J. Lam, "Asynchronous output-feedback stabilization of discrete-time Markovian jump linear systems", in Proceedings of the 51st IEEE Conference on Decision and Control, Hawall, USA, Dec. 2012, pp. 1307-1312.

[17] Ü. Y. Ogras, and R. Marculescu, "Analysis and optimization of prediction-based flow control in networks-on-chip", ACM Transactions on Design Automation of Electronic Systems, vol. 13, no. 1, article 11, 2008.

[18] U. Shaked, "Improved LMI representations for the analysis and the design of continuous-time systems with polytopic type uncertainty", IEEE Transactions on Automatic Control, vol. 46, no. 4, pp. 652-656, 2001.

[19] Y. Jia, "Alternative proofs for improved LMI representations for the analysis and the design of continuous-time systems with polytopic type uncertainty: A predictive approach", IEEE Transactions on Automatic Control, vol. 48, no. 8, pp. 1413-1416, 2003.

[20] K. M. Grigoriadis, and R. E. Skelton, "Low-order control design for LMI problems using alternating projection methods", Automatica, vol. 32, no. 8, pp. 1117-1125, 1996.

[21] H. K. Tam, and J. Lam, "Robust deadbeat pole assignment with gain constraints: An LMI optimization approach", Optimal Control Applications and Methods, vol. 21, no. 6, pp. 243-256, 2000.

[22] H. Su, M. Z. Q. Chen, J. Lam, and Z. Lin, "Semi-global leader-following consensus of linear multi-agent systems with input saturation via low gain feedback", IEEE Transactions on Circuits and Systems I, vol. 60, no. 7, pp. 1881-1889, 2013.

[23] J. Xiong, J. Lam, H. Gao, and D. W. C. Ho, "On robust stabilization of Markovian jump systems with uncertain switching probabilities", Automatica, vol. 41, no. 5, pp. 897-903, 2005.

[24] J. Xiong, and J. Lam, "Robust $\mathrm{H}_{2}$ control of Markovian jump systems with uncertain switching probabilities", International Journal of Systems Science, vol. 40, no. 3, pp. 255-265, 2009.

[25] G. Wang, and S. Xu, "Robust $H_{\infty}$ filtering for singular time-delayed systems with uncertain Markovian switching probabilities", International Journal of Robust and Nonlinear Control, vol. 25, no. 3, pp. 376-393, 2015 . 
[26] O. L. V. Costa, J. B. R. Do Val, and J. C. Geromel, "A convex programming approach to $\mathrm{H}_{2}$ control of discrete-time Markovian jump linear systems", International Journal of Control, vol. 66, no. 4, pp. 557579, 1997.

[27] Z. Shu, J. Lam, and J. Xiong, "Static output-feedback stabilization of discrete-time Markovian jump linear systems: A system augmentation approach", Automatica, vol. 46, no. 4, pp. 687-694, 2010.

[28] M. Shen, C. C. Lim, and P. Shi, "Reliable $H_{\infty}$ static output control of linear time-varying delay systems against sensor failures", International Journal of Robust and Nonlinear Control, vol. 27, no. 16, pp. 3109 3123, 2017.

[29] J. Song, Y. Niu, and Y. Zou, "Asynchronous output feedback control of time-varying Markovian jump systems within a finite-time interval", Journal of the Franklin Institute, vol. 354, no. 15, pp. 6747-6765, 2017.

[30] L. Zhang, P. Shi, C. Wang, and H. Gao, "Robust $H_{\infty}$ filtering for switched linear discrete-time systems with polytopic uncertainties", International Journal of Adaptive Control and Signal Processing, vol. 20, no. 6, pp. 291-304, 2006.

[31] O. L. V. Costa, M. D. Fragoso, and M. G. Todorov, "A detector-based approach for the $\mathrm{H}_{2}$ control of Markov jump linear systems with partial information", IEEE Transactions on Automatic Control, vol. 60, no. 5, pp. 1219-1234, 2015.

[32] $\mathrm{H}$. $\mathrm{Li}$, and $\mathrm{M}$. Fu, "A linear matrix inequality approach to robust $H_{\infty}$ filtering", IEEE Transactions on Signal Processing, vol. 45, no. 9, pp. 2338-2350, 1997.

[33] R. Orsi, U. Helmke, and J. B. Moore, "A Newton-like method for solving rank constrained linear matrix inequalities", Automatica, vol. 42, no. 11, pp. 1875-1882, 2006.

[34] R. Orsi, LMIRank: software for rank constrained LMI problems, http://users.cecs.anu.edu.au/〜robert/lmirank/, 2005.

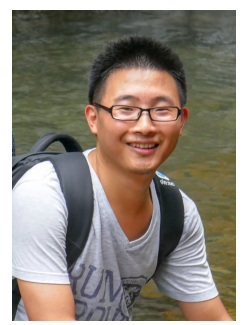

Jun Song received his B.E. degree in Electronic Science and Technology in 2011 and M.E. degree in Pattern Recognition and Intelligent System in 2014, both from Anhui University, Hefei, China. He is now pursuing his Ph.D. degree in Control Science and Engineering at East China University of Science and Technology, Shanghai, China.

From December 2015 to February 2016 and July 2016 to August 2016, he was a Research Assistant in the Department of Mechanical Engineering, the University of Hong Kong, Hong Kong, China. From October 2016 to October 2017, he was a Visiting Ph.D. Student with the Department of Computer Science, Brunel University London, Uxbridge, U.K.. His research interests including Markovian jump systems and optimal control, stochastic systems, finite-time stability, and sliding mode control.

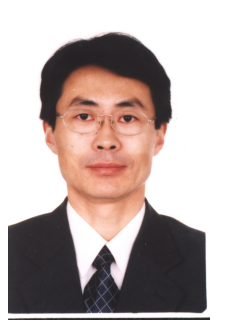

Yugang Niu received his $\mathrm{MSc}$ and $\mathrm{PhD}$ degrees from the Nanjing University of Science \& Technology in 1992 and 2001, respectively. In 2003, he joined the School of Information Science \& Engineering, East China University of Science \& Technology and is currently a professor

Now, he is Associate Editor of several international journals, including INFORMATION SCIENCES, NeURoComputing, IET CONTROL THEORY \& APPLICATIONS, JOURNAL OF THE FRANKLIN INSTITUTE, and INTERNATIONAL JOURNAL OF SYSTEM SCIENCES. He is also a member of Conference Editorial Board of IEEE Control Systems Society. His research interests include stochastic systems, sliding mode control, Markovian jump systems, networked control systems, wireless sensor network, Smart grid.

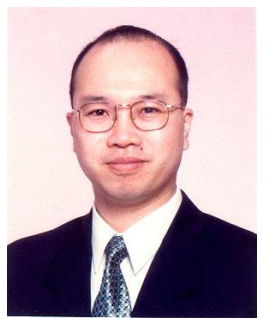

James Lam received the B.Sc. (Hons.) degree in Mechanical Engineering from the University of Manchester, and the M.Phil. and Ph.D. degrees from the University of Cambridge. He was a Lecturer with the City University of Hong Kong and the University of Melbourne. He joined University of Hong Kong in 1993, where he is currently the Chair Professor of Control Engineering. He is a Croucher Scholar, Croucher Fellow, and Distinguished Visiting Fellow of the Royal Academy of Engineering. He received the Ashbury Scholarship, the A.H. Gibson Prize, and the H. Wright Baker Prize for his academic performance.

Professor Lam is a Chartered Mathematician, Chartered Scientist, Chartered Engineer, Fellow of Institute of Electrical and Electronic Engineers, Fellow of Institution of Engineering and Technology, Fellow of Institute of Mathematics and Its Applications, and Fellow of Institution of Mechanical Engineers. He is Editor-in-Chief of IET CONTROL THEORY AND APPLICATIONS and JOURNAL of The FrankLin Institute, Subject Editor of Journal of Sound AND VibRATION, Editor of ASIAN JOURNAL OF CONTROL, Senior Editor of COGENT ENGINEERING, Associate Editor of AUtomaticA, InTERnATIONal Journal of Systems SCIENCE, MULTidimensional Systems AND Signal Processing, and Proc. IMechE Part I: Journal of Systems AND CONTROL ENGINEERING. He is a member of the IFAC Technical Committee on Networked Systems, and Engineering Panel (Joint Research Schemes), Research Grant Council, HKSAR. His research interests include model reduction, robust synthesis, delay, singular systems, stochastic systems, multidimensional systems, positive systems, networked control systems and vibration control. He is a Highly Cited Researcher in Engineering (Thomson Reuters, 2014, 2015, 2016) and Computer Science (Thomson Reuters, 2015).

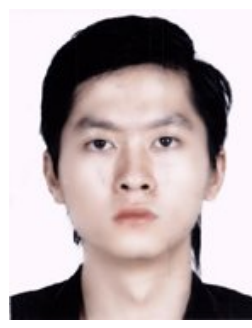

Zhan Shu was born in Nanchang, Jiangxi province, China, in 1982. He received his B.Eng. degree in Automation from Huazhong University of Science and Technology (HUST) in 2003, and the Ph.D. degree in Control Engineering from The University of Hong Kong (HKU) in 2008. From 2008 to 2009, he worked in $\mathrm{HKU}$ as a research assistant/associate. From 2009 to 2011, he was a postdoctoral researcher in the Hamilton Institute, National University of Ireland, Maynooth. He joined the University of Southampton, U.K., in 2011, taking a lectureship in the Faculty of Engineering and the Environment. He is a Member of IEEE and an invited reviewer of MATHEMATICAL REVIEW of the American Mathematical Society. He serves as an Associate Editor for MATHEMATICAL Problems in Engineering, an Associate Editor for Asian Journal of CONTROL, an Associate Editor for JoURnAL of THE FRANKLIN INSTITUTE, and a member of the IEEE CONTROL SySTEMS Society Conference EDITORIAL BOARD. His current research interests include hybrid systems, stochastic systems, positive systems, delay systems, robust control, decentralized control, estimation and filtering, model free control, control application in energy systems, systems biology with emphasis on immunology. 